\title{
A New Scheme To Enhance The Performance Of Permutation Index-Differential Chaos Shift Keying Communications System
}

Nizar Albassam ( $D$ nazarhooby@yahoo.co.uk)

Middle East College https://orcid.org/0000-0001-6642-9174

Oday Al-Jerew

Asia Pacific International College

\section{Research}

Keywords: Differential Coherence, AWGN Channel, Differential Correlator, Permutation Index

Posted Date: March 15th, 2021

DOl: https://doi.org/10.21203/rs.3.rs-296095/v1

License: (c) (i) This work is licensed under a Creative Commons Attribution 4.0 International License.

Read Full License 


\title{
A New Scheme To Enhance The Performance Of Permutation Index-Differential Chaos Shift Keying Communications System
}

\author{
Nizar Al Bassam ${ }^{1 *}$ and Oday Al-Jerew ${ }^{1,2}$
}

\footnotetext{
${ }^{*}$ Correspondence:

nazarhooby@yahoo.co.uk

${ }^{1}$ Department of Electronics and

Communications Engineering,

Middle East College, Muscat,

Oman

Full list of author information is

available at the end of the article
}

\begin{abstract}
In this paper, a new scheme based on permutation index-differential chaos shift keying is proposed, modeled, and evaluated in AWGN channel environment. Data is sent by frames, each frame is headed by a single reference signal and followed by some information bearing signals. Modulation is performed through permutations of a reference signal according to the mapped data. At the receiver, each incoming information bearing signal undergoes all inverse permutation possibilities to perform a correlation with the delayed and stored version of the received reference signal. To decode the information bits, the detector selects the highest correlator outputs. The proposed scheme named Single

Reference-Permutation Index-Differential Chaos Shift Keying (SR-PI-DCSK) is an enhanced version of PI-DCSK, and uses a single reference signal for multiple information bearing ones. Hence, the energy requirement is saved by almost a half. The bit error performance is studied using the baseband system model and analytically tested using Gaussian Approximation (GA) method. Results show the BER performance outperforms other standard and recently developed differentially coherent chaos systems, including Permutation Index-DCSK by an average of $2.25 \mathrm{db}$. Moreover, the analytical form which is developed to predict the Bit Error Rate (BER) is validated by simulation. Results demonstrate the performance in AWGN is closely matching with the simulation results, particularly at high SNR.
\end{abstract}

Keywords: Differential Coherence; AWGN Channel; Differential Correlator; Permutation Index

\section{Introduction}

Chaotic communication systems are broadly classified into coherent, non-coherent and differentially coherent systems [?][?]. Implementation of coherent systems requires an accurate generation of the chaotic segment at the receiver which is identical to the transmitted one. Due to noise contribution, the received signal becomes distorted and difficult to synchronize. Theoretical implementation of coherent Chaos Shift Keying (CSK) is proposed in [?]. Additionally, the maximum likelihood detection scheme is used in [?] to enhance Bit-Error-Rate (BER) performance for multiple users. Changing of Probability Density Function (PDF) for the chaotic source to match PDF of the channel to enhance BER is simulated and discussed in [?]. All the previous coherent systems require an accurate regeneration of the chaotic segment at the receiver which is difficult to achieve due to the chaotic nature of the source and the channel noise. The work in [?][?] consider demodulation 
in non-coherent systems based on the received signal properties (i.e. bit energy, map estimation, or time reversing). These systems require an effective extracting of the transmitted signal and precise threshold optimization between signal elements. In all differential chaos based scheme, data symbols is presenting by sending the reference signal followed by information bearing as in DCSK. At the receiver, correlation is performed by multiplying the incoming information bearing signal with the delayed version of the reference signal. In Correlation Delay Shift Keying (CDSK), the transmitted signal consists of the current information signal added to the reference signal of the previous symbol as discussed in [?][?][?][?]. However, the proposed schemes are complex and require additional synchronization circuits. In DCSK, the reference signal and information bearing signal are sent through consecutive time slots. Therefore, a frequency delay line is required at the receiver. Radio Frequency (RF) delay line removal to reduce systems complexity in DCSK receiver is suggested in [?] and developed in [?]. The removal is done by sending the reference signal and the information bearing signal on the same time slot and separate them using Walsh code. Here, more delay elements are added to the transmitter in addition to the usage of lengthy Walsh code. Reference and information signal separation using initial condition modulation is examined in [?]. Efficient utilization of the transmitted signal energy using Multicarrier Modulation for DCSK (MC-DCSK) is introduced in [?]. However, a hardware implementation requires a bank of narrow-band modulators with a high degree of accuracy in the design to maintain subcarrier synchronization. The work in [?] uses Space-Time Block Code DCSK (STBC-DCSK) to enhance BER performance. Although STBC-DCSK enhances the noise performance of DCSK, the data rate and the average energy for each bit are similar to that in DCSK with an additional STBC encoder at the transmitter.

Permutation index DCSK is used to build the first MARY modulation scheme in [?][?], an advanced version of the PI-DCSK is proposed on [?]. Improved versions of PI-DCSK where reference chaotic segment and information bearing signal are combined on one-bit duration are presented in [?]. Walsh Code is also recalled in [?] to separate reference and information bearing signal. While the dual used mode of the reference signal is being suggested in [?]. In all the previous literature, DCSK is based on having one to one relationship between the reference signal and information bearing signal. In this brief, a common reference signal is used to modulate multiple information bits respective by signal permutation according to the information bits, hence the proposed system enhances the energy efficiency by almost $50 \%$. Also, it offers double bit rates compared to standard chaos-based systems namely DCSK, Correlation Delay Shift Keying, High Efficiency-DCSK (HEDCSK), and Time Reversal-DCSK(TR-DCSK).

The main contribution of this paper as follows: we develop an SR-PI-DCSK which uses a single reference signal for multiple information bearing ones. we compare BER performance with the other differentially and permutation based DCSK that uses a dedicated reference signal for each information symbol. The developed model is mathematically analyzed and verified by simulation under various factors and different channel conditions.

The remainder of this paper is organized as follows. Section 2 and Section 3 present the transmitted signal frame and scheme for the proposed SR-PI-DCSK system, 
respectively. The details of system analysis are discussed in Section 4 and Section 5 . The details of experimental results with discussion are provided in Section 6. Finally, Section 7 concludes the paper.

\section{System Signal Format}

The transmitted signal frame of SR-PI-DCSK is shown in Fig. 1. Each frame consists of a reference signal segment with a length of $\beta$ samples followed by $L$ information bearing signals. Information bearing signal is nothing but only a permutated version of the reference signal. Permutation order is determined by the information bits with the length of $n$, for example, to send 2 information bits, we need to set up $2^{2}$ permutations from the reference signal while 3 bits requires 9 different permutations.

\section{The Proposed Scheme}

To generate the signal in Fig. 1, the design of modulator and demodulator of SRPI-DCSK system are shown in Fig. 2 and Fig. 3, respectively. A reference signal $X$ is generated with the chaotic samples having a length of $\beta$. This reference signal is sent initially prior to the frame through switch $\mathrm{S}$, to modulate the first information symbol, the same version of the reference signal is delayed and undergoes permutation operation according to the transmitted bits. The reference signal and its permutated version are related in a way that ensures very low correlation such that $X P_{j}^{T}(X)=0[?]$, where $P_{j}($.$) is the permutation operator. A chaotic signal$ with the spreading factor of $\beta$ can be permutated in $\beta !-1$ permutation. Hence for information bits vector $a=\left(\begin{array}{llll}a_{1} & a_{2} & \ldots & a_{n}\end{array}\right)$ with length of $n$, we will select one distinguished permutation out of $\beta$ ! -1 and to have permutation mapping such away that $a_{j} \rightarrow P_{j}$ and $P_{j} \in\left\{\begin{array}{llll}P_{1} & P_{2} \ldots P_{2^{n}}\end{array}\right\}$. Furthermore, $P_{j}$ is the permutation performed in the chaotic sequence to present $a_{j}$ data set. Thus, the first transmitted frame can be given by the baseband representation as:

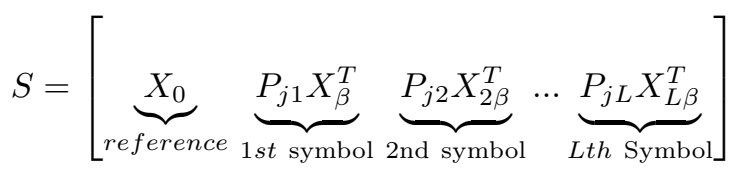

Where $X_{0}=\left(\begin{array}{llll}x_{1} & x_{2} & \ldots & x_{\beta}\end{array}\right)$ is the reference chaotic segment and $P_{j 1} X_{\beta}^{T}$ represents the first information signal which is nothing but reference signal delayed by $\beta$ and permutated by $P_{j 1}$ mapped from information vector $a_{j 1}$. Moreover, $L$ is the frame length. In this paper, the analysis is simplified using the analysis of the first symbol only, similar methods can be applied on other following information symbols since all the symbols are statistically independent.

Under the assumption that the transmitted signal is received via AWGN channel and the transmitter and receiver are perfectly synchronized. The following operations are performed in the receiver: (i) the first incoming reference signal, is loaded into delay block and stored for correlation with other $L$ symbol as shown in Fig. 2. (ii) the following information bearing signal is directly loaded to the permutation block to perform all possible $M$ inverse permutations, where $M=2^{n}$.

To detect the first information symbol, the received reference signal is delayed by $\beta$, (i.e $X_{\beta}+W_{\beta}$ ) is correlated with output of $m^{t h}$ inverse permutation block output 
where $m \in(1,2, \ldots, M)$, hence, the output can be written as $P_{m}^{-1}\left(P_{j 1} X_{\beta}+W_{0}\right)$ where $W_{0}=\left(\begin{array}{llll}w_{1} & w_{2} & \ldots & w_{\beta}\end{array}\right)$ is the noise vector and $w_{k}$ is AWGN noise sample having power spectral density of $N_{0} / 2$. Therefore, the $m^{\text {th }}$ correlator output can be found as

$$
\begin{aligned}
D_{m} & =\left(X_{\beta}+W_{\beta}\right) P_{m}^{-1}\left(P_{j 1} X_{\beta}+W_{0}\right) \\
& =\underbrace{X_{\beta} P_{m}^{-1} P_{j 1} X_{\beta}^{T}}_{S S \text { or SI }}+\underbrace{X_{\beta} W_{0}^{T}+W_{\beta} P_{m}^{-1} P_{j 1} X_{\beta}^{T}}_{S N}+\underbrace{W_{\beta} P_{m}^{-1} W_{0}^{T}}_{N N}
\end{aligned}
$$

Where $S S$ represents desired signal component at $m=j_{1}$, while $S I$ is the inter signal interference components at $m \neq j_{1}$. Inter signal interference results from the correlation between the chaotic signal and its permutated version. The $N N$ is the noise to noise correlation that has a significant impact on the performance particularly at a large spreading factor of $\beta$.

The first estimated permutation index $\hat{j}_{1}$ is determined by correlator which is having the maximum output compared with the others, hence

$$
\hat{j}_{1}=\arg \max \left\{\mathrm{D}_{m}\right\}, m \in\left\{1,2, \cdots 2^{n}\right\}
$$

By estimating the correct $\hat{j}_{1}$, the transmitter can predict which permutation which has been used to send the information.

\section{System Analysis}

The BER performance analysis is performed analytically using GA methods that are suitable for a system having large spreading factors, However, for low spreading factors, an accurate integration method is suggested [?].

For each $L$ symbols sent, one refence signal is dedicated, therefore, $L E_{\text {symbol }}=$ $(L+1) E_{s}$, where $E_{\text {symbol }}$ is the average symbol energy while $E_{s}$ is average segment energy given by $\beta V(X)$ and $V($.$) is the variance operator. For standard DCSK$ and CDSK and standard scheme [?][?][?][?][?], each information bearing signal is accompanied by reference signal. Therefore to send $L$ symbols, we need to have $L$ additional reference signal, hence energy and bandwidth efficiency are almost doubled. Compared with the proposed scheme, and for sufficient value of $L$, the energy efficiency can be calculated as

$$
E_{\text {efficiency }}=\frac{L+1}{2 L} \approx 50 \% \text { for sufficient value of } L
$$

Since each symbol is carrying $n$ bits, hence average bit energy is given by

$$
\begin{aligned}
& L E_{\text {symbol }}=L n E_{b} \\
& \frac{(L+1)}{L n} \beta V(X)=E_{b}
\end{aligned}
$$




\section{Performance analysis}

In this section, the performance analysis of the proposed scheme is analyzed in AWGN channel environment to predict the BER performance. The chaotic sequence is generated from the symmetric Tent map, given by the equation $x_{k+1}=1-2\left|x_{k}\right|$. The chaotic sequence $x$ is having uniform distribution between -1 and 1 with zero mean and computed variance $V(x)=1 / 3$ and $V\left(x^{2}\right)=4 / 45=4 / 5 V^{2}(x)$ [?]. For the rest of the analysis, the following assumptions are considered (i) The correlation between the chaotic sequence and its permutated version is decaying quickly for a sufficient correlation window. (ii) The correlation between the chaotic sequence and the noise sample is statistically independent.

The Bit Error Probability of the proposed scheme is determined by calculating (i) errorness of permutation estimation $P r_{\text {map }}$, (ii) errorness probability of bits detection which largely depends on the number of bits $n$ which calculated by the following

$$
P_{r_{m a p}}=\frac{2^{(n-1)}}{2^{n}-1} P_{r_{e d}}
$$

Correct estimation of the transmitted sequence is done by selecting the maximum absolute value of one of the correlator outputs. This output results from the correlation between the delayed reference signal and inverse permutated information bearing signal. Each correlator output can be modeled as a Gaussian random variable $D_{m}$. For equiprobable transmitted sequence, the error probability of permutation index estimation conditioned by $P_{j}$ is given by

$$
P_{\text {red }}=\left(P_{r}\left|D_{j 1}\right|<\max P_{r}\left|D_{m}\right|\right) \text { for } 1 \leq \mathrm{m} \leq 2^{n}, m \neq j_{1}
$$

Where the $D_{j 1}$ and $D_{m}$ are decision variable at $m^{t h}$ and $j_{1}^{t h}$ correlator output. The error will occur only if any value of $D_{m}$ can have a magnitude larger than $D_{j 1}$. To detect the first information bearing signal, the output of $m^{\text {th }}$ correlator can have two values and can be rewritten as

$$
\mathrm{D}_{\mathrm{m}}= \begin{cases}S S+S N+N N & m=j_{1} \\ S I+S N+N N & m \neq j_{1} .\end{cases}
$$

The correlation components can be calculated as

$$
\begin{aligned}
& S S=X_{\beta} P_{j_{1}}{ }^{-1} P_{j_{1}} X_{\beta}=X_{\beta} X_{\beta}{ }^{T}=\sum_{k=1}^{\beta} x_{k} x_{k} \\
& S N=X_{\beta} W_{0}+W_{\beta} P_{j_{1}}{ }^{-1} P_{j_{1}} X=\sum_{k=1}^{\beta} x_{k-\beta} w_{k}+\sum_{k=1}^{\beta} w_{k-\beta} x_{k}
\end{aligned}
$$




$$
N N=W_{\beta} P_{j_{1}}^{-1} W_{0}=\sum_{k=1}^{\beta} w_{k-\beta} w_{k}^{\prime},
$$

where $w_{i}^{\prime}$ is the permutated noise sample.

Based on the assumption that the correlation between noise sample $w_{i}$ and chaotic samples $x_{j}$ for all $i$ and $j$ is negligible, for sufficient value of $\beta$, then

$$
E\left(D_{j_{1}}\right)=E(S S)+\mathrm{E}(\mathrm{SN})+\mathrm{E}(\mathrm{NN})=\mathrm{E}\left(\sum_{k=1}^{\beta} x_{k} x_{k}\right)+0+0=\beta V(x)=\frac{L}{L+1} n E_{b}
$$

$$
V(S S)=V\left(\sum_{k=1}^{\beta} x_{k} x_{k}\right)=V\left(\sum_{k=1}^{\beta} x^{2}{ }_{k}\right)=\frac{4}{5} \beta V(x) V(x)=\frac{4(L)^{2}}{5 \beta(L+1)^{2}} n^{2} E_{b}^{2}
$$

$$
V(\mathrm{SN})=2 \beta V(x) V(w)=2 \frac{L n}{L+1} E_{b} N_{0}
$$

$$
V(\mathrm{NN})=V(w) \cdot V\left(w^{\prime}\right)=\frac{\beta}{4} N_{o}^{2} .
$$

While $E\left(D_{m}\right) \approx 0$, similarly

$$
\begin{aligned}
V\left(D_{m}\right) & =V(S I)+V(\mathrm{SN})+V(\mathrm{NN}) \\
& =\frac{(L)^{2} n^{2}}{\beta(L+1)^{2}} E_{b}^{2}+2 \frac{L n}{(L+1)} E_{b} N_{0}+\frac{\beta}{4} N_{o}^{2}
\end{aligned}
$$

The output of each $2^{n}-1$ correlators $D_{m}$ for $m \neq j_{1}$ are statistically independent random values characterized by a normal distribution with zero mean and can be given as

$$
f_{D_{m}}(y)=\frac{1}{\sqrt{2 \pi V\left(D_{m}\right)}} e^{-\frac{(y)^{2}}{2 V\left(D_{m}\right)}}
$$

While the correlator output conditioned by correct permutation can be given as

$$
f_{D_{j_{1}}}(y)=\frac{1}{\sqrt{2 \pi V\left(D_{j_{1}}\right)}} e^{-\frac{\left(y-E\left(D_{j_{1}}\right)\right)^{2}}{2 V\left(D_{j_{1}}\right)}}
$$


It is easier to calculate the probability of correct permutation which is occurred only if magnitude of $D j_{1}>D_{1}, D j_{1}>D_{2}$ and $\ldots D j_{1}>D_{M}$, therefore the probability of correct map detection can be given as [?]

$$
p_{\text {map }}=1-\int_{0}^{\infty} F_{D m}(y)^{2^{n}-1} f_{j_{1}}\left(D_{j_{1}}\right)(y) d y
$$

where $F_{D m}(y)$ is the commutative distribution function given by

$$
F_{D_{m}}(y)=\operatorname{erf}\left(\frac{y}{\sqrt{2 \pi V\left(D_{m}\right)}}\right)
$$

The overall BER of the system can be given by substitution on

$$
\begin{aligned}
B E R_{S R-P I-D C S K} & =\frac{2^{(n-1)}}{2^{n}-1}\left[1-\frac{1}{\sqrt{2 \pi \frac{4(L)^{2} n^{2}}{5 \beta(L+1)^{2}} E_{b}^{2}+2 \frac{L n}{L+1} E_{b} N_{0}+\frac{\beta}{4} N_{o}^{2}}}\right. \\
& \int_{0}^{\infty} \operatorname{erf}\left(\frac{y}{\sqrt{2 \pi \frac{(L)^{2} n^{2}}{\beta(L+1)^{2}} E_{b}^{2}+2 \frac{L n}{L+1} E_{b} N_{0}+\frac{\beta}{4} N_{o}^{2}}}\right)^{2^{n}-1} \\
& \left.e^{-\frac{\left(y-E\left(D_{j_{1}}\right)\right)^{2}}{2 V\left(D_{j_{1}}\right)}} d y\right]
\end{aligned}
$$

\section{Experimental results}

In this section, different standard chaos schemes including the recently proposed scheme have been simulated and compared with the suggested system in AWGN channel environment and with various $E_{b} / N_{0}$. Furthermore, the proposed system has been compared with the standard PI-DCSK [?]. Analytic performance based on Eq. 23 has been compared with simulation results for different frame lengths. Finally, the effect of the spreading factors on the proposed scheme has been examined.

In Fig. 4, the SR-PI-DCSK is compared with the DCSK, CDSK, HE-DCSK, and TR-DCSK. The system outperforms the other systems by an average of $2 \mathrm{~dB}$ except for DCSK which exceeds the suggested system by $1 \mathrm{~dB}$, although the proposed system offers a double bit rate. This is due to non-complete orthogonality at spreading factor $\beta=100$. When the spreading factor is increased to $\beta=150$, it can be easily noted that the SR-PI-DCSK system performance enhanced by an average of $3 \mathrm{~dB}$ for all other systems as shown in Fig. 5. Obviously, the superiority in the performance continue while spreading factor increased to $\beta=200$ and $\beta=300$ as shown in Fig. 6 and Fig. 7, respectively. This can be explained by the fact that, compared to other system chaos signal, the average transmitted signal energy per bit for the proposed system has been saved by $2 L /(L+1) \approx 50 \%$ compared to the standard systems to which corresponds to a gain or horizontal displacement of BER. In addition to the very low cross correlation value at the correlator output between the interference component. 
Comparison of the SR-PI-DCSK system performance at various frame length has been demonstrated on Fig. 8, Fig. 9, Fig. 10 and Fig. 11. The comparison is performed at the increment trend of the spreading factor. It is obvious that the performance of the proposed system with frame length $L=3$ is better than systems with the length of $L=2$ and $L=1$ or PI-DCSK [?]. It evident that the performance is degraded at a high value of $\beta$ due to noise to noise ( $N N$ on Eq. 12) which at the correlator output.

A theoretical estimation of BER for the proposed system is developed using GA and validated using computer simulation. Excellent agreement between theoretical expression in Eq. 23 and simulation results that shown in Fig. 12, Fig. 13, Fig. 14 and Fig. 15 for $\beta=100, \beta=150, \beta=300$ and $\beta=500$, respectively. The results obtained clearly validate the expression derived.

Effect of increasing of spread factor $\beta$ has been studied and illustrated in Fig. 16 at fixed value of $E_{b} / N_{0}$ (at $13 \mathrm{~dB}$ and $17 \mathrm{~dB}$ ). It is clearly shown that the performance is enhanced as $\beta$ increases and reach its ideal performance at $\beta=100$, and this matched with the results shown in Fig. 4, Fig. 5, Fig. 6, and Fig. 7. After $\beta$ reach 100 , the performance starts to decrease with $\beta$ due to dominance of noise to noise expression $(N N)$ at high spreading factor as illustrated by the equation (23)

\section{CONCLUSION}

A new permutation based DCSK system named Single Reference-Permutation Index-Differential Chaos Shift Keying (SR-PI-DCSK) is proposed, designed, and validated through simulation. A theoretical formula to predict the bit error rate is developed and verified. The proposed system sends information symbols in a frame, each frame consists of a reference signal followed by a sequence of $L$ information bearing signal. This reduces the energy requirement and enhanced the BER. The BER performance is simulated and compared with DCSK, CDSK, HE-DCSK, and PI-DCSK schemes. Results illustrate the system outperform other chaos based systems, particularly at large spreading factor. Comparison results illustrate the advantage of the proposed scheme specifically at moderate $E_{b} / N_{0}$ over the other schemes. Based on the GA method, theoretical prediction for BER is developed and compared with the simulation results. Excellent matching between the derived form and simulation results is noticed.

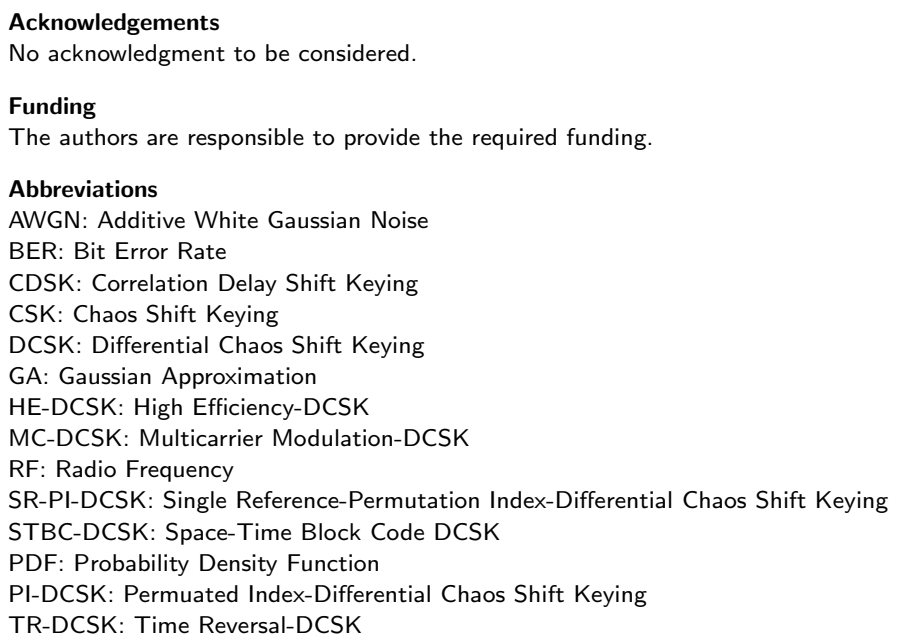


Availability of data and materials

All data and materials are generated based on simulation.

Competing interests

The authors declare that they have no competing interests.

Authors' contributions

Al Bassam conceived of the presented the SR-PI-DCSK scheme. develop the theoretical model, perform the mathematical analysis and simulation Al-Jerew perform and validate the simulation results with the theoretical model, verify result consistency and contribute to the research literature section. Both authors discussed the results and contributed to the final manuscript.

Author details

${ }^{1}$ Department of Electronics and Communications Engineering, Middle East College, Muscat, Oman. ${ }^{2}$ Business Information Systems, Asia Pacific International College, Sydney, Australia.

References

Figures

Figure 1 SR-PI-DCSK transmitted signal frame.

Figure 2 SR-PI-DCSK transmitter block diagram.

Figure 3 SR-PI-DCSK receiver diagram.

Figure 4 The bit error rate performance of SR-PI-DCSK verses DCSK, CDSK, HE-DCSK, and TR-DCSK for $\beta=100$.

Figure 5 The bit error rate performance of SR-PI-DCSK verses DCSK, CDSK, HE-DCSK, and TR-DCSK for $\beta=150$.

Figure 6 The bit error rate performance of SR-PI-DCSK verses DCSK, CDSK, HE-DCSK, and TR-DCSK for $\beta=200$.

Figure 7 The bit error rate performance of SR-PI-DCSK verses DCSK, CDSK, HE-DCSK, and TR-DCSK for $\beta=300$.

Figure 8 The bit error rate performance at different frame length $(L=1, L=2$ and $L=3)$ for SR-PI-DCSK for $\beta=50$. 
Figure 9 The bit error rate performance at different frame length $(L=1, L=2$ and $L=3)$ for SR-PI-DCSK for $\beta=100$.

Figure 10 The bit error rate performance at different frame length $(L=1, L=2$ and $L=3)$ for SR-PI-DCSK for $\beta=150$.

Figure 11 The bit error rate performance at different frame length $(L=1, L=2$ and $L=3)$ for SR-PI-DCSK for $\beta=300$.

Figure 12 Theoretical and simulation bit error performance for SR-PI-DCSK for different frame length for $\beta=100$. Simulation results are depicted by lines while theoretical results are depicted by markers.

Figure 13 Theoretical and simulation bit error performance for SR-PI-DCSK for different frame length for $\beta=150$. Simulation results are depicted by lines while theoretical results are depicted by markers.

Figure 14 Theoretical and simulation bit error performance for SR-PI-DCSK for different frame length for $\beta=300$. Simulation results are depicted by lines while theoretical results are depicted by markers.

Figure 15 Theoretical and simulation bit error performance for SR-PI-DCSK for different frame length for $\beta=500$. Simulation results are depicted by lines while theoretical results are depicted by markers.

Figure 16 Theoretical and simulation bit error performance for SR-PI-DCSK at different spreading value and $L=3$. 

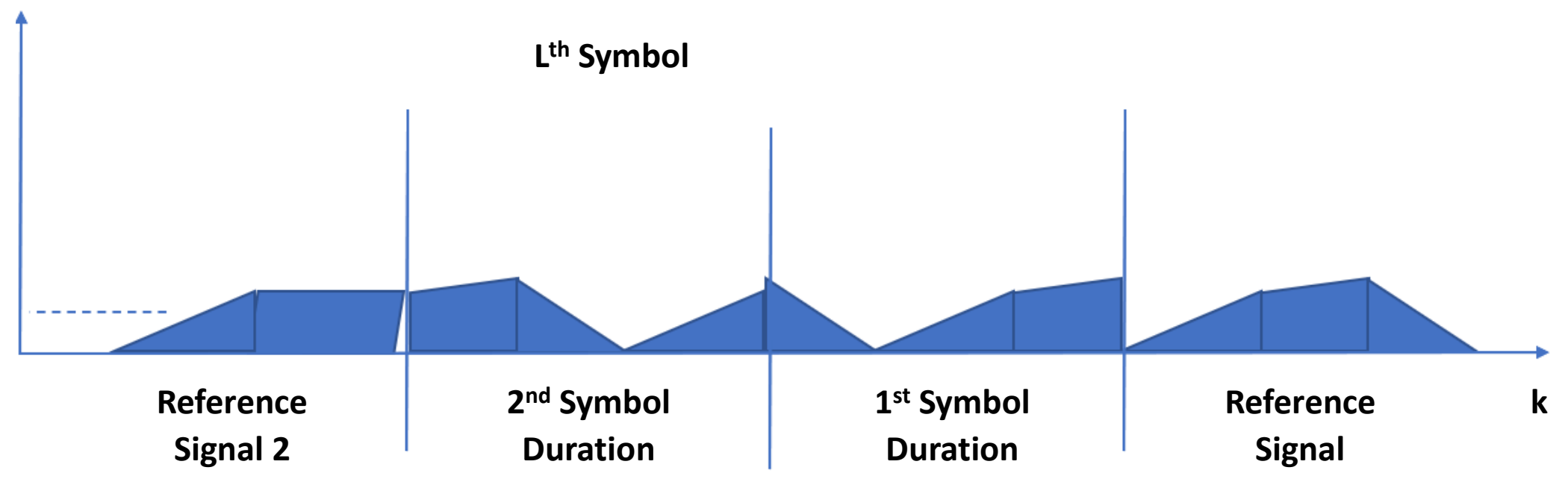


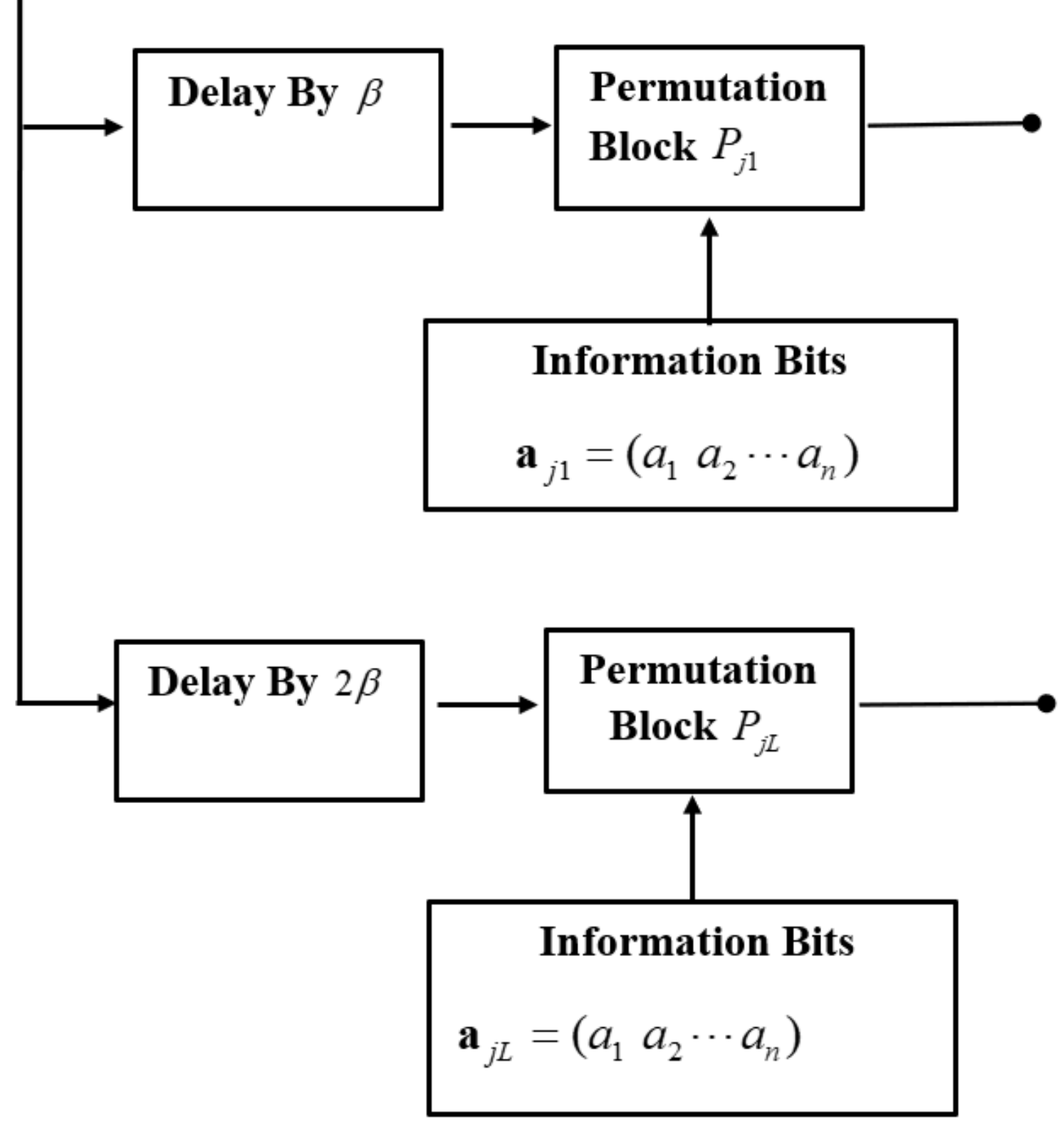

Transmitted Frame 


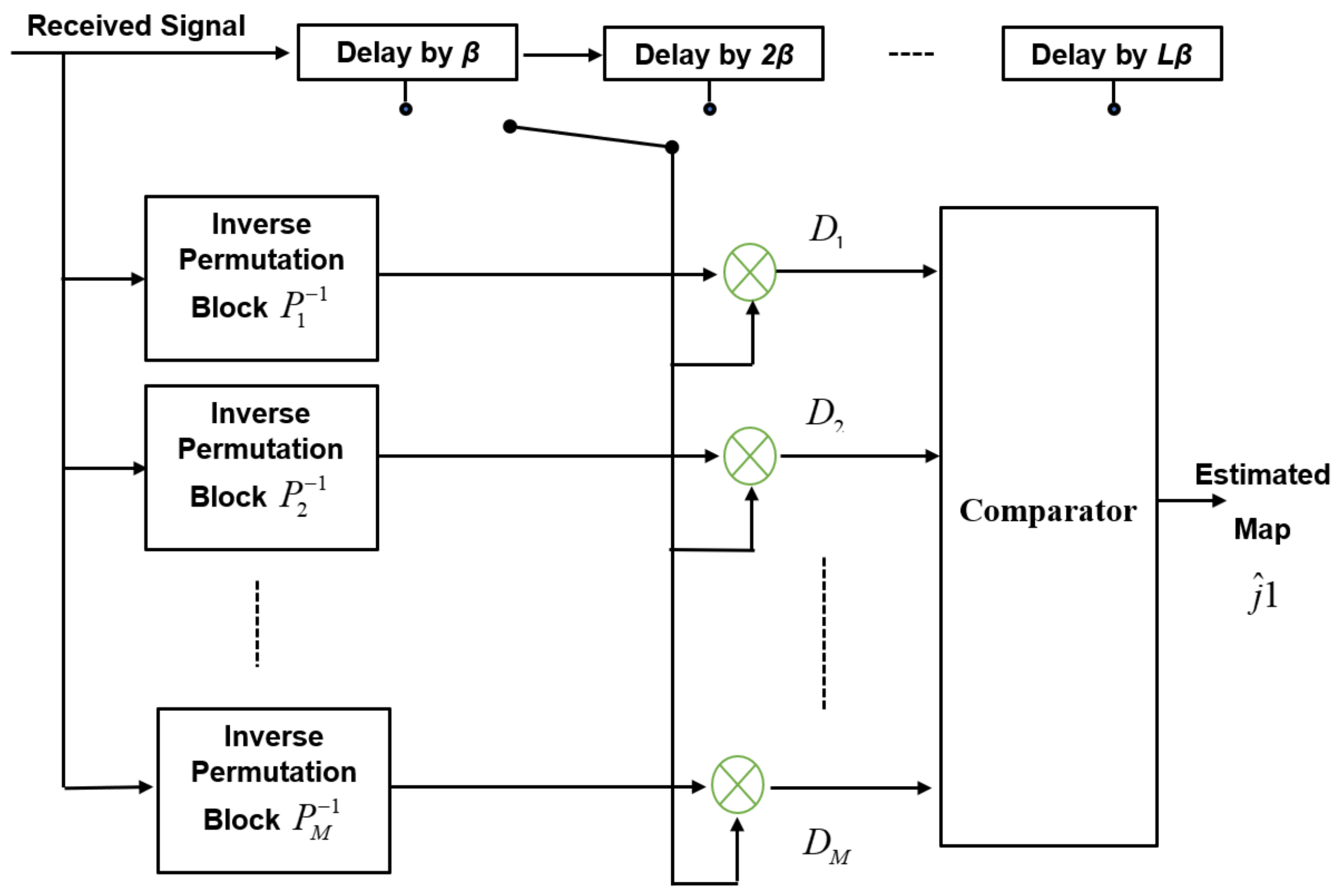




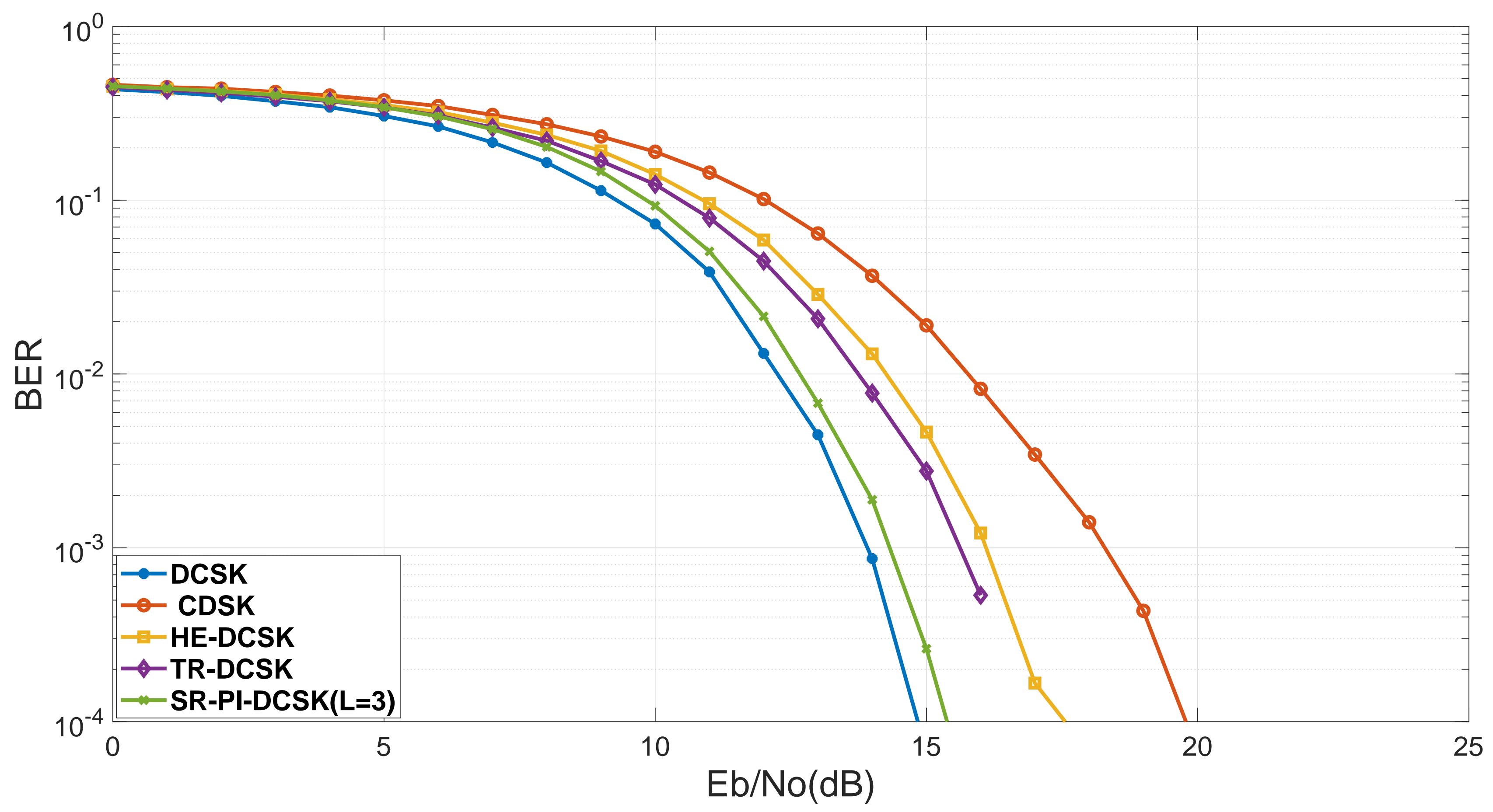




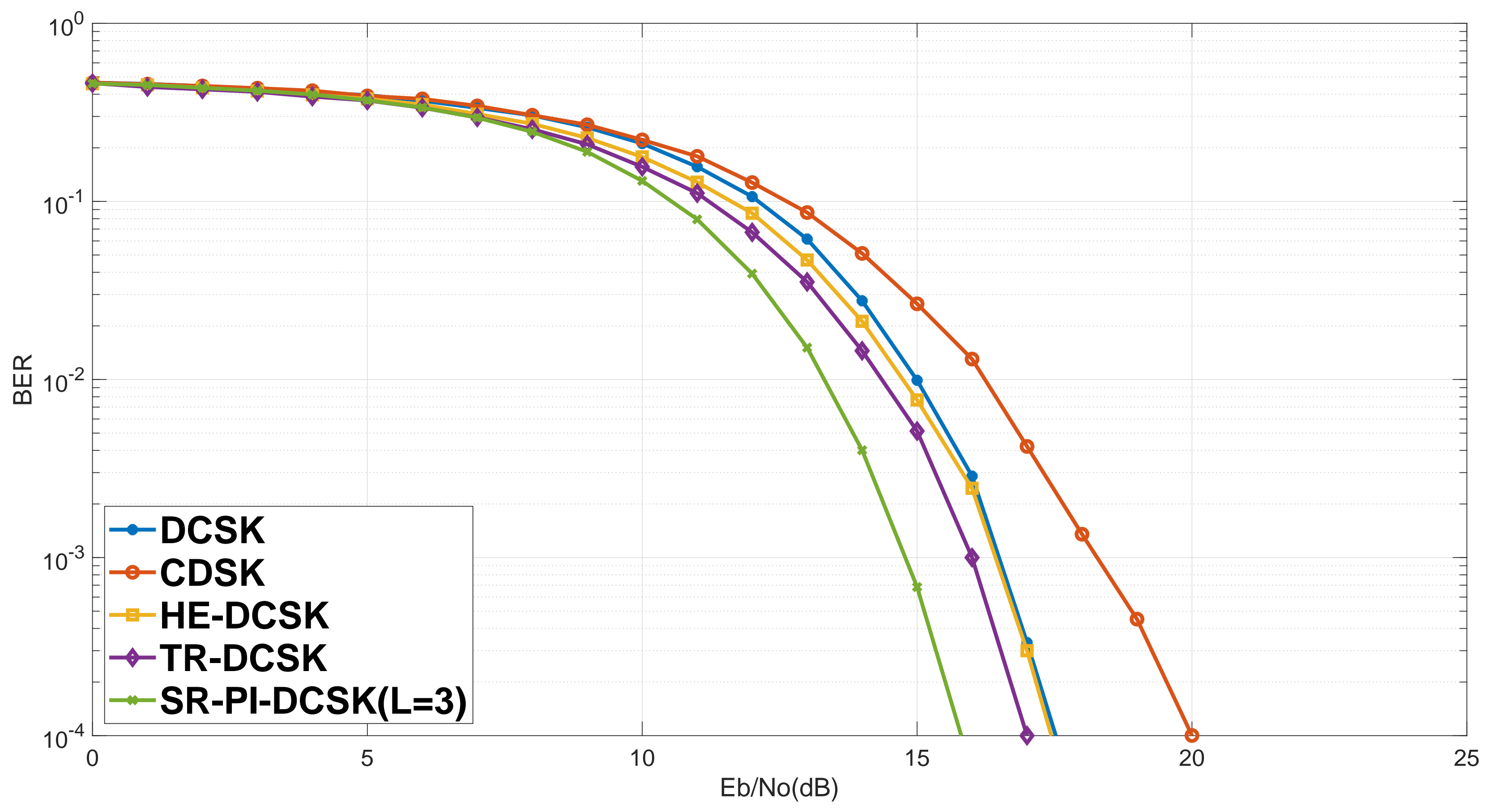




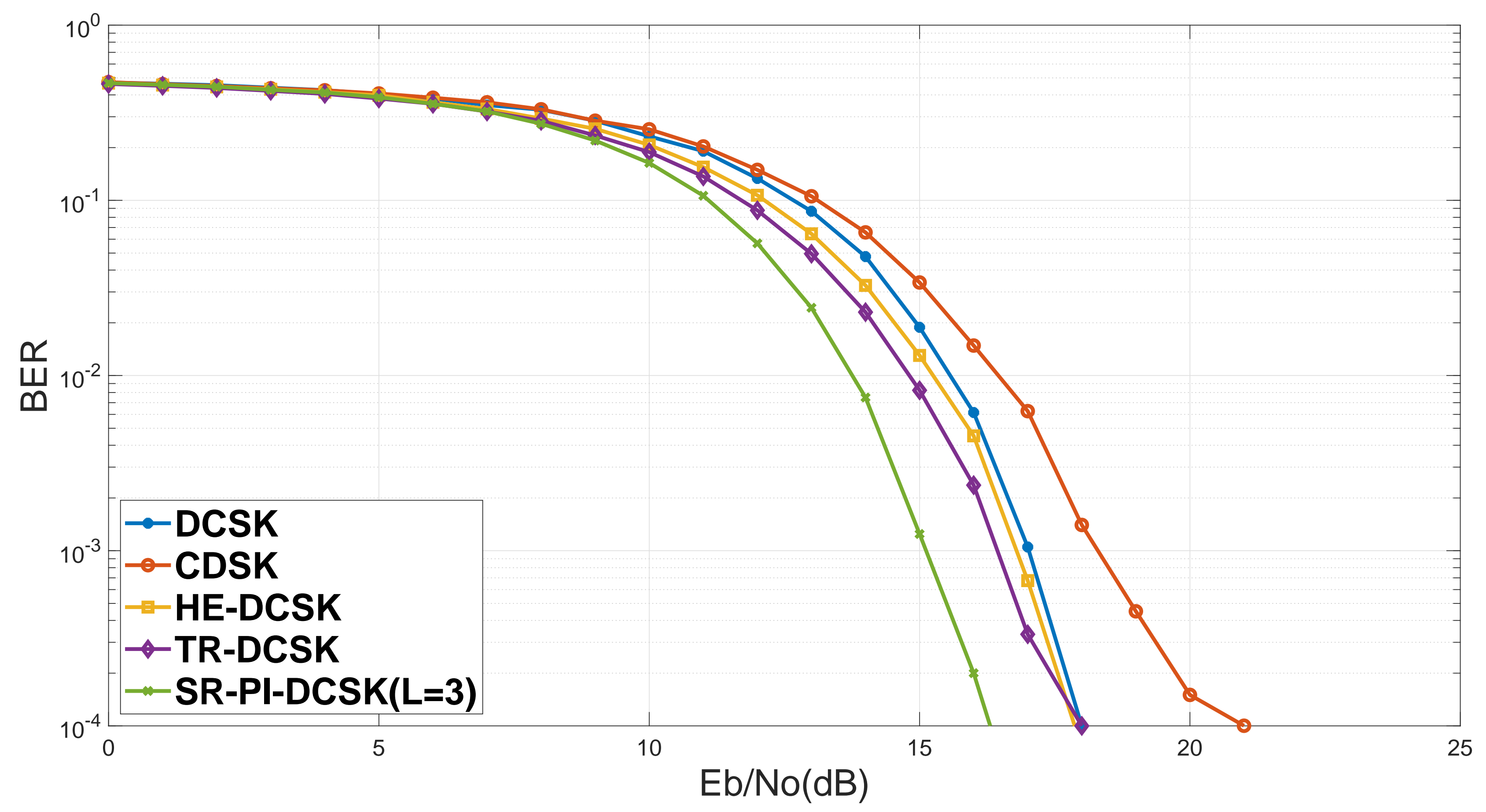




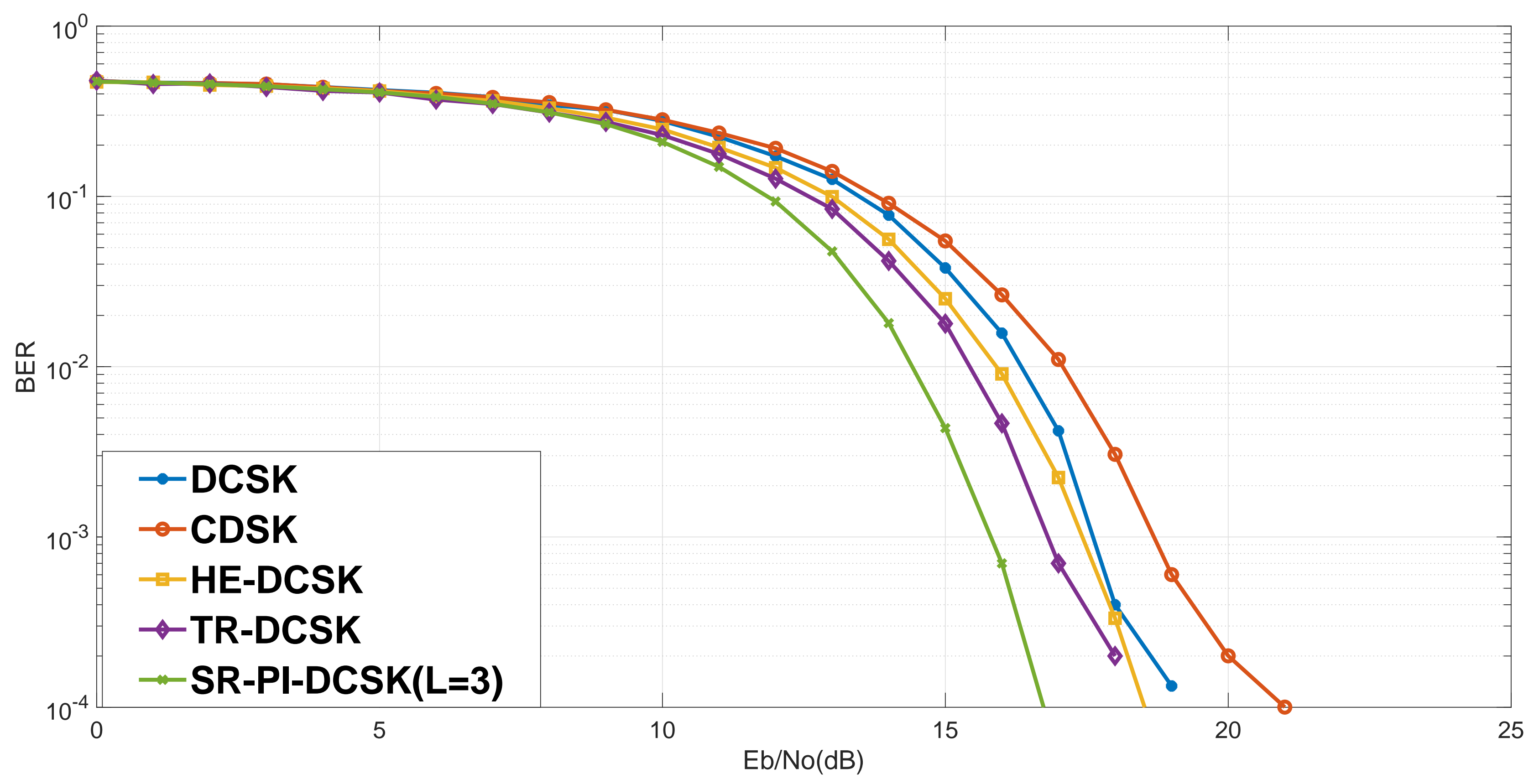




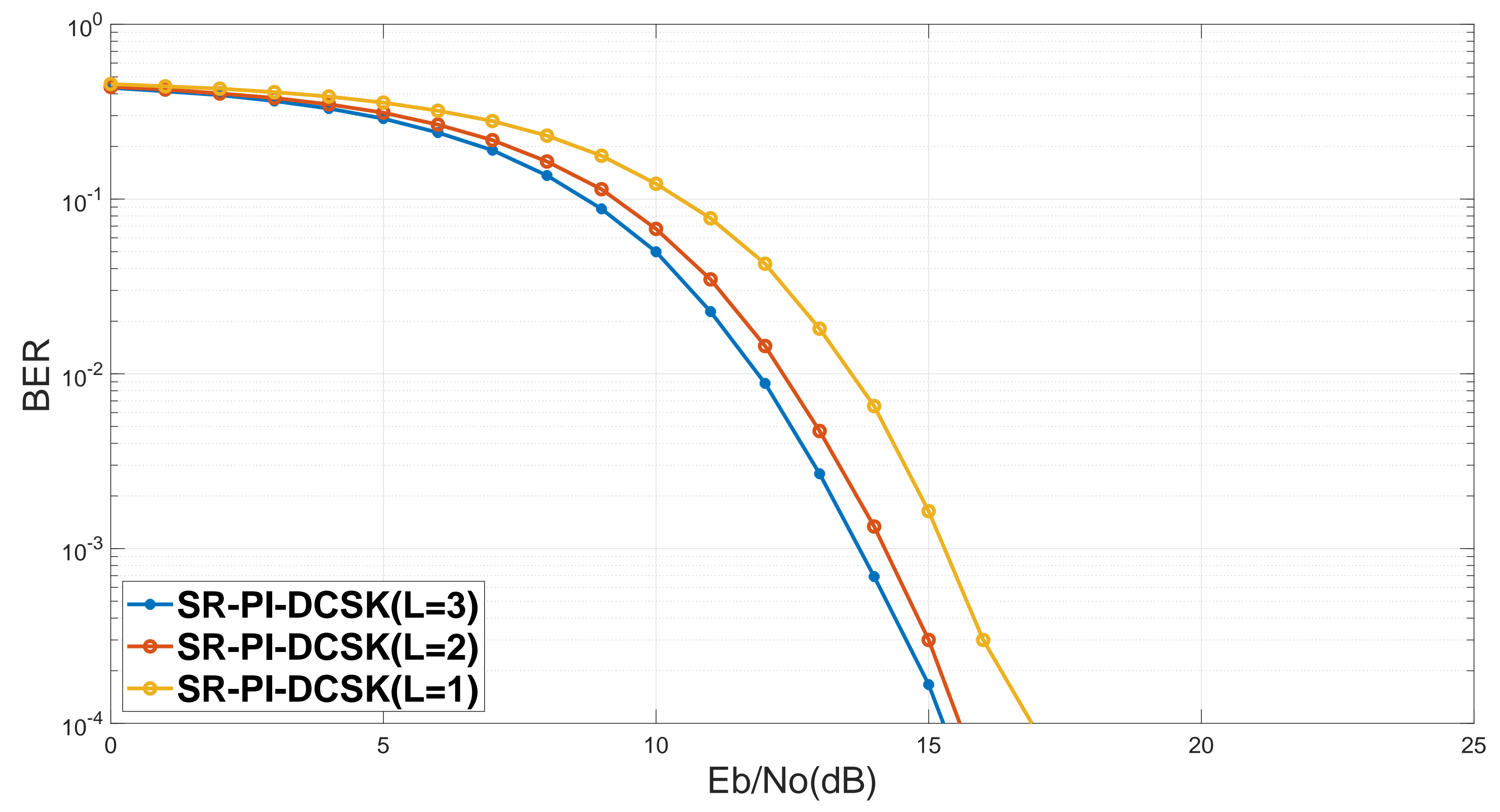




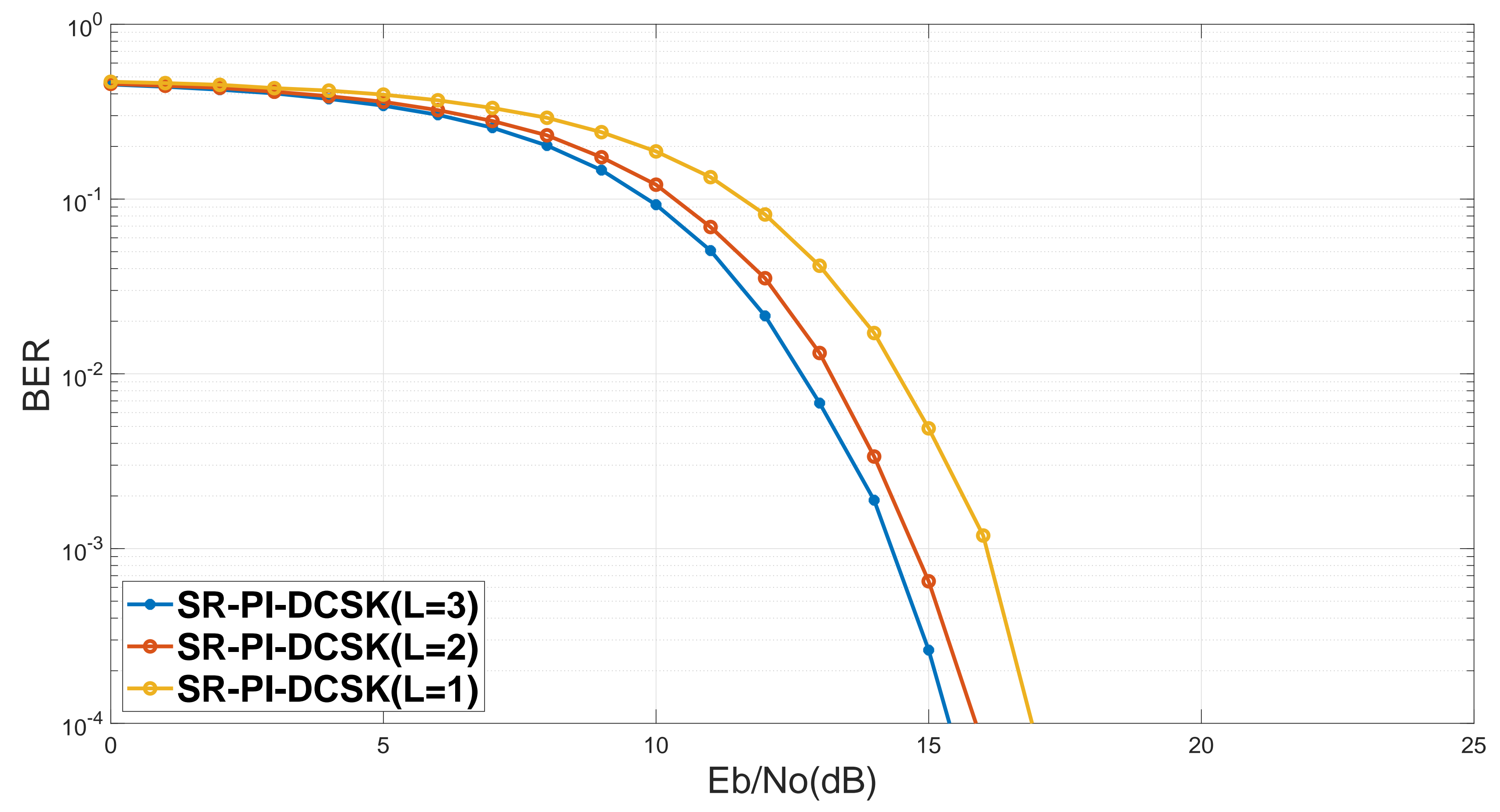




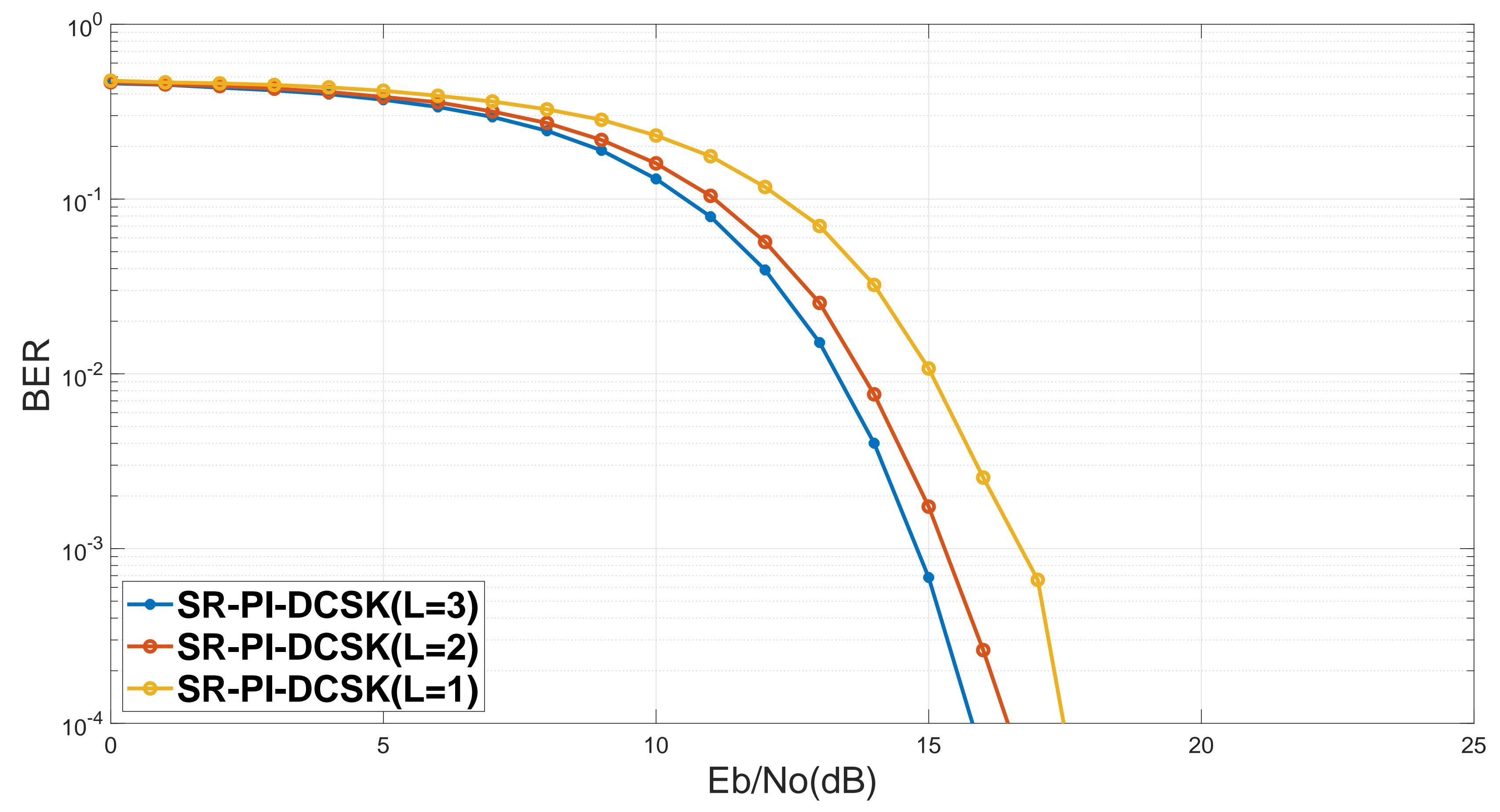




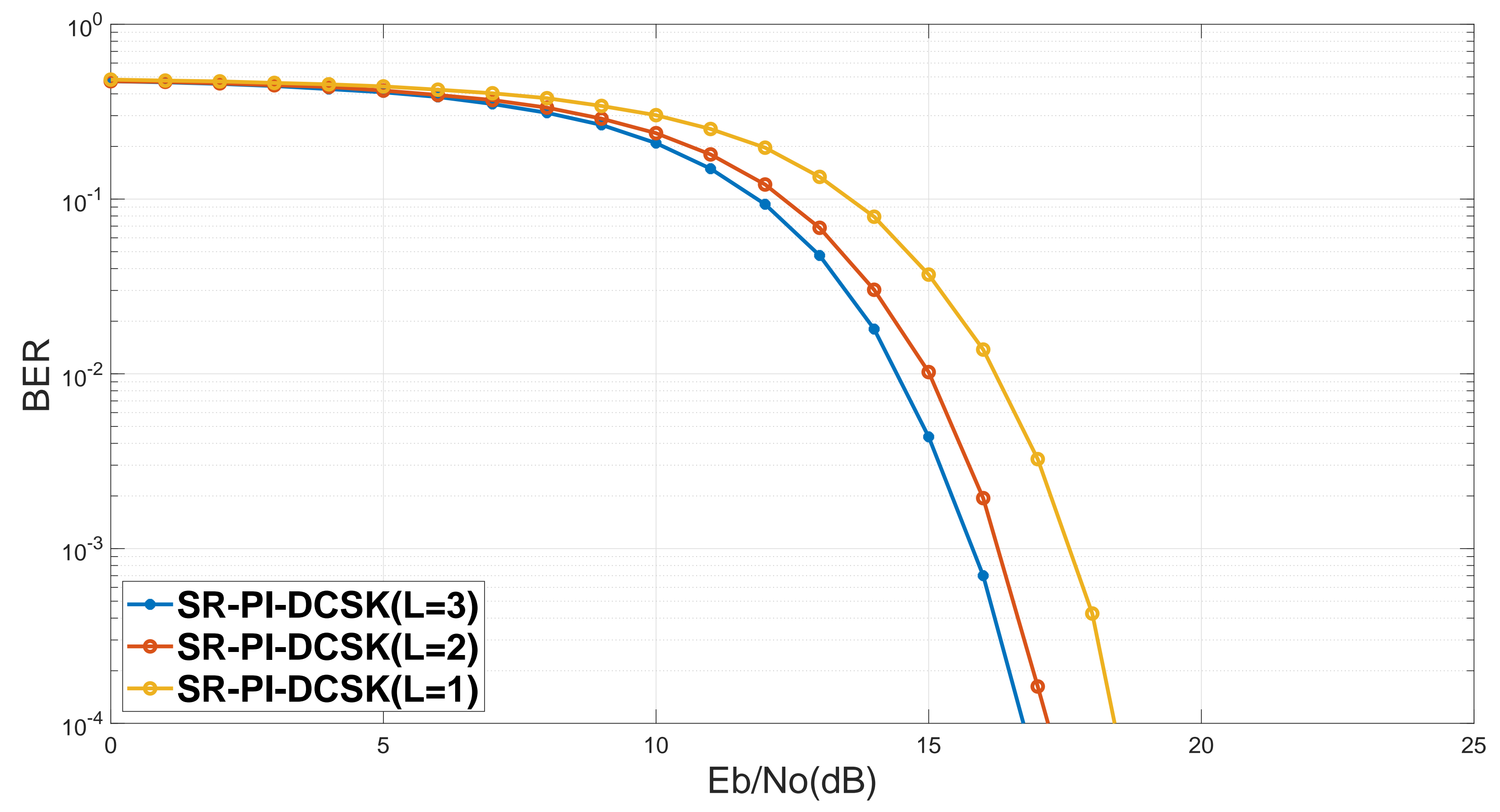




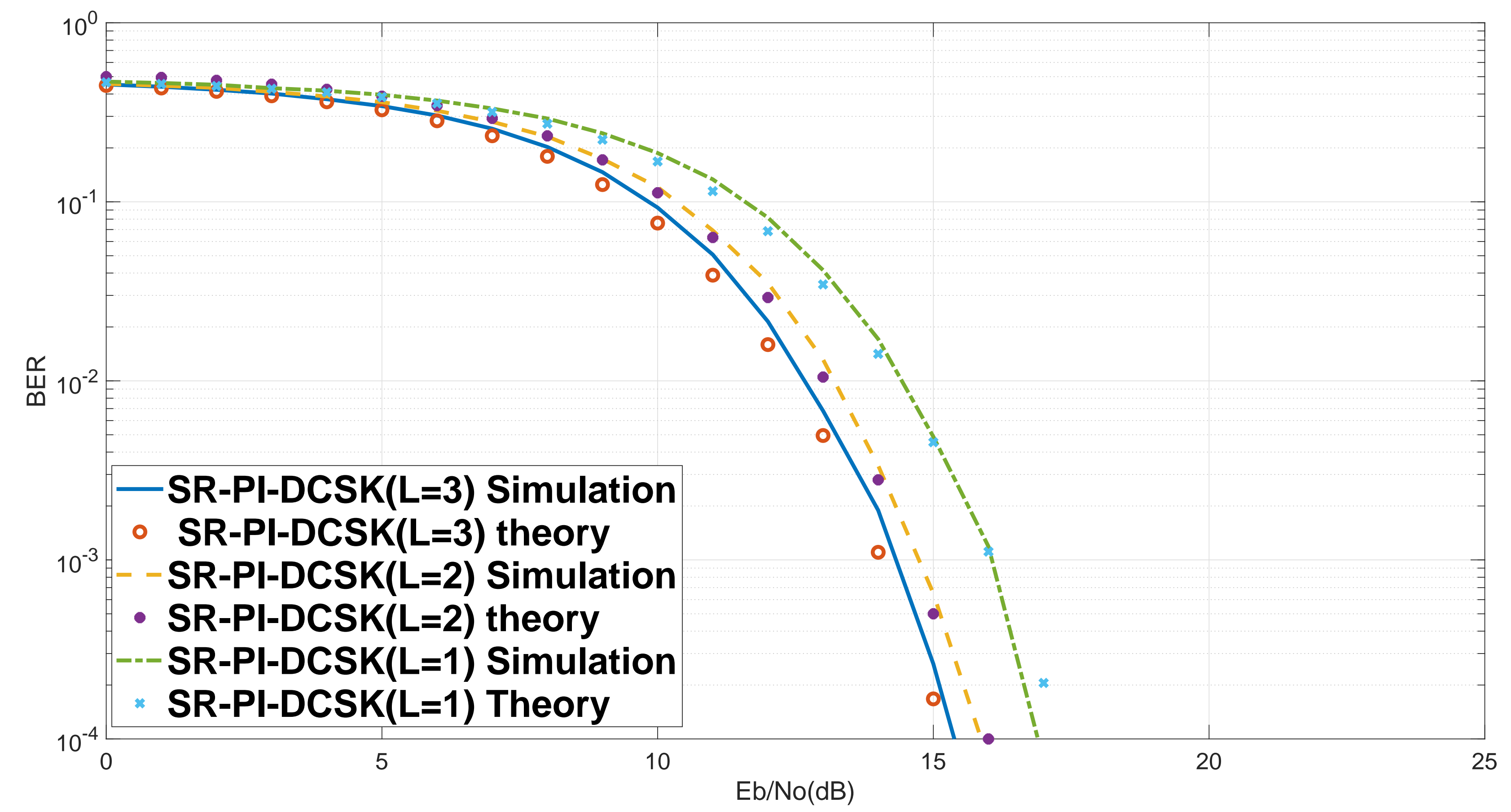




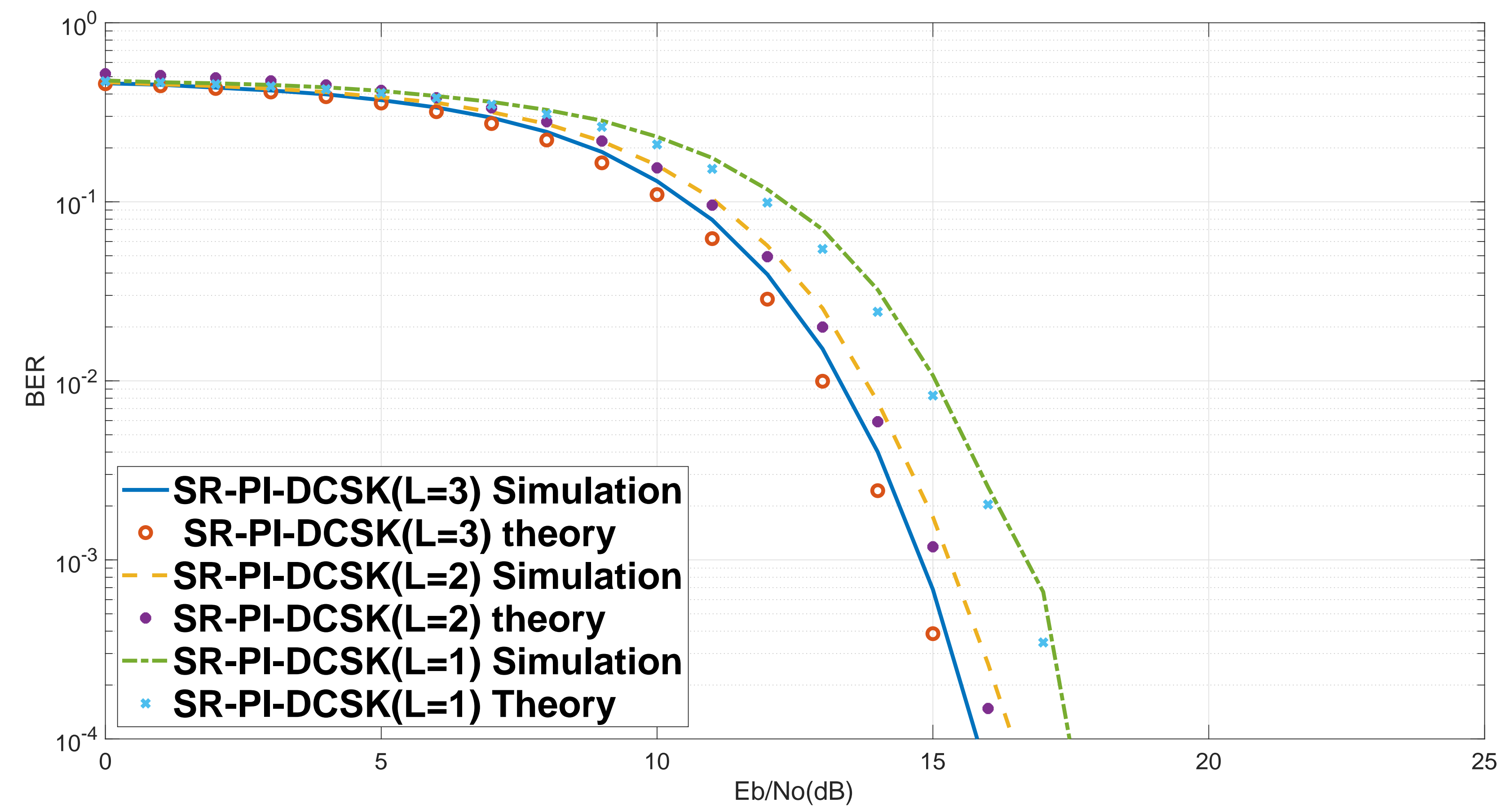




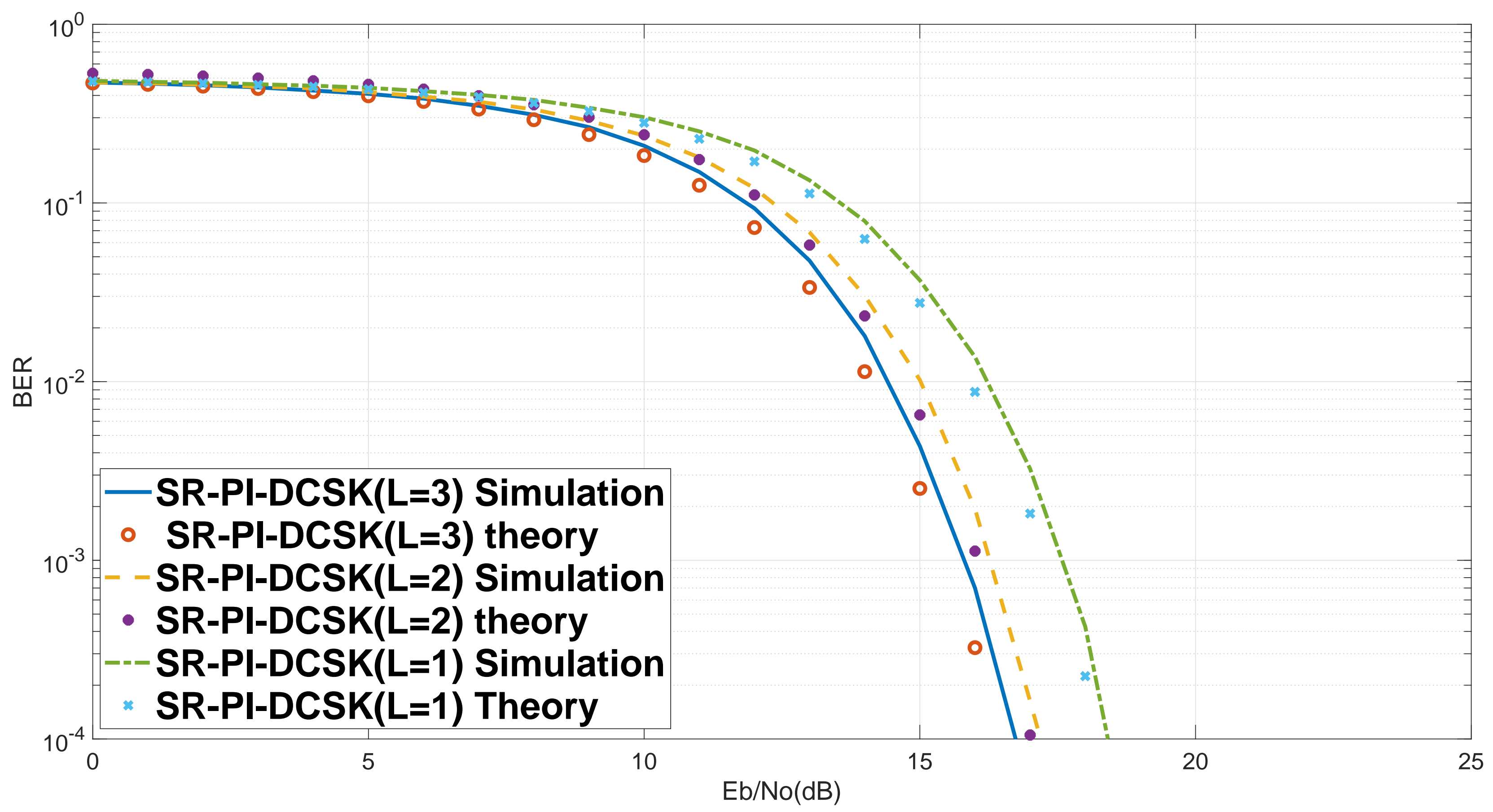




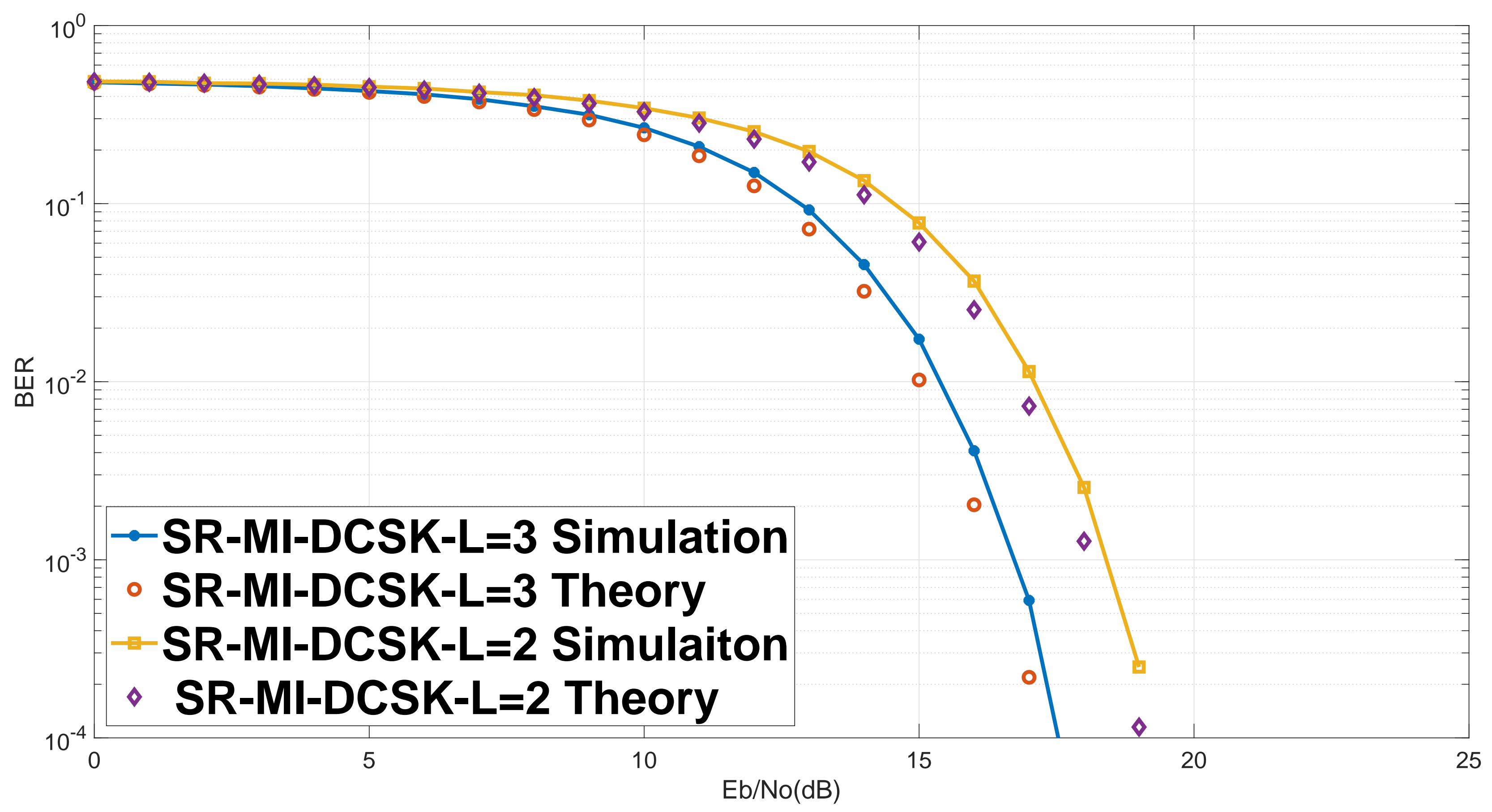




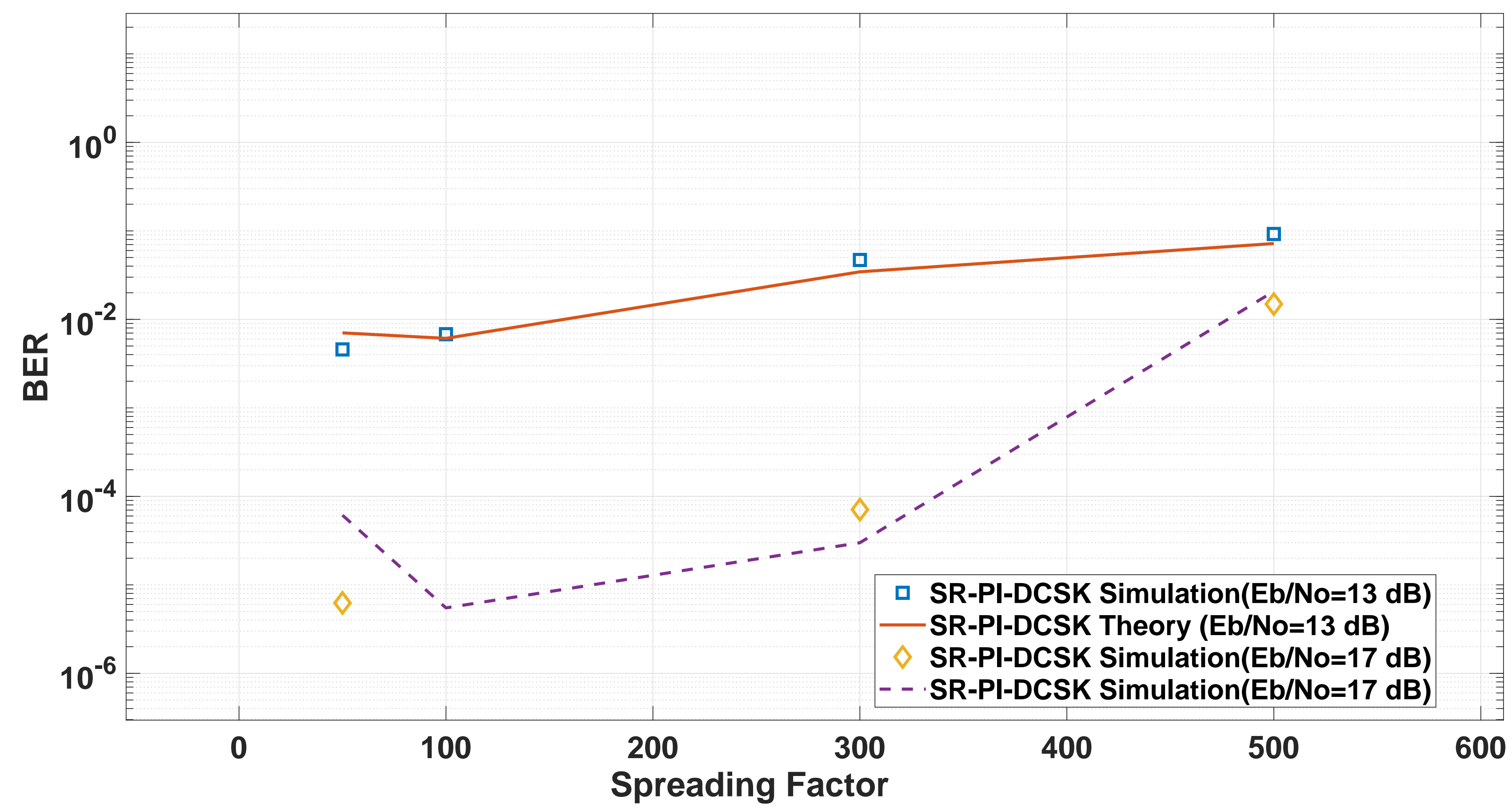


Figures

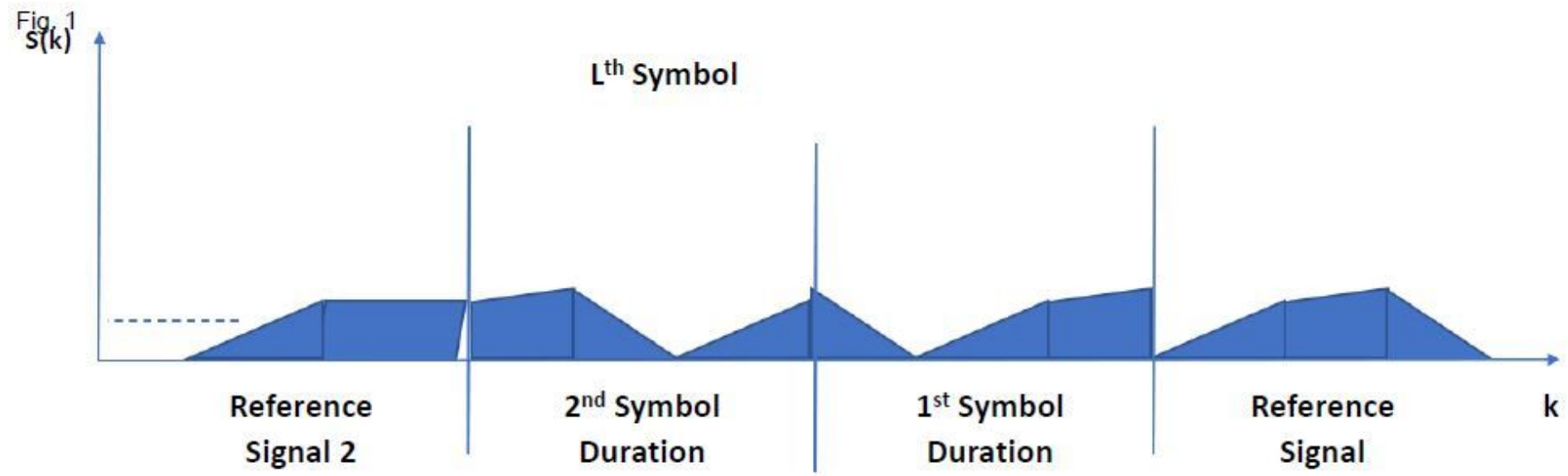

Figure 1

SR-PI-DCSK transmitted signal frame.

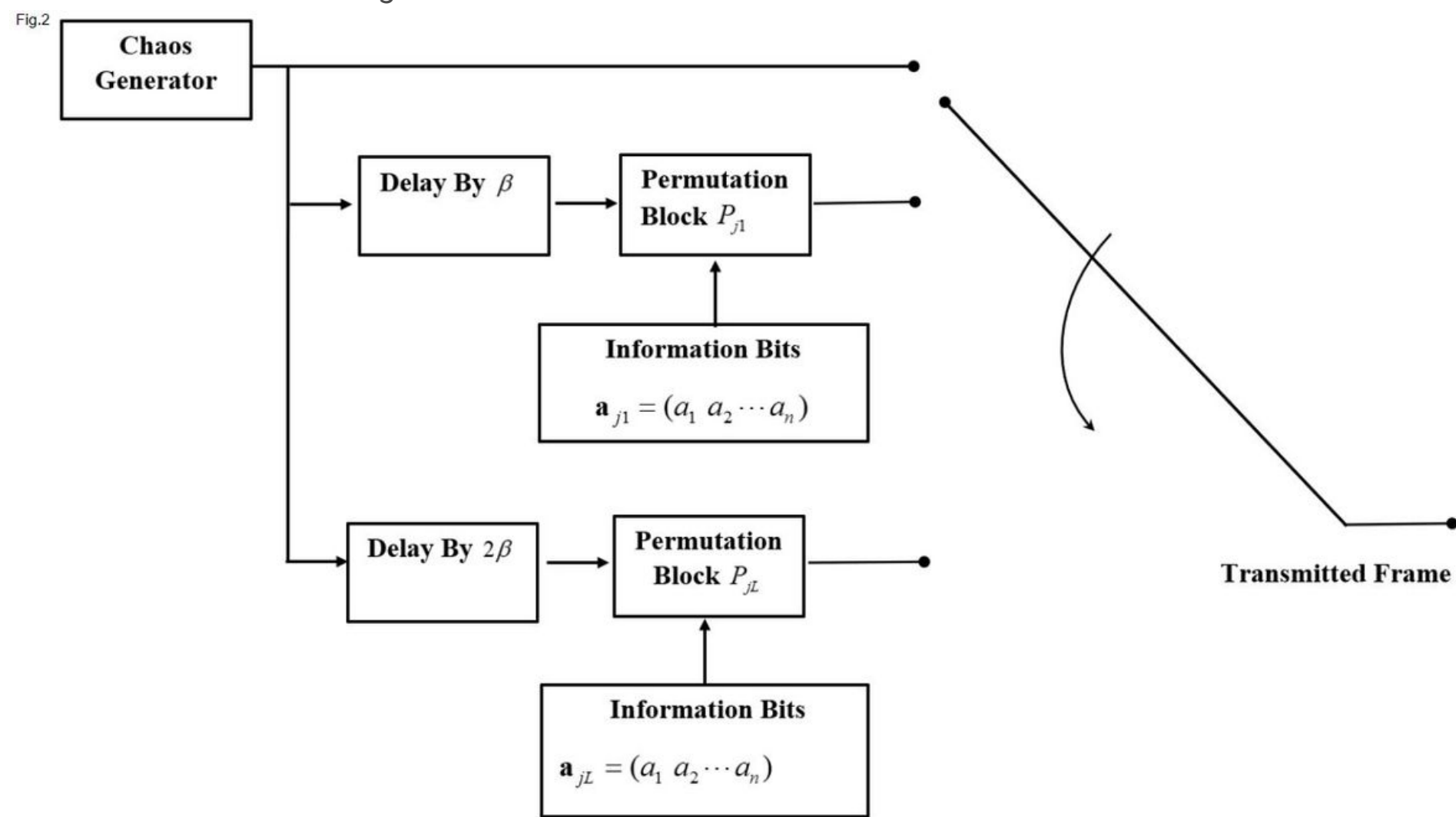

Figure 2

SR-PI-DCSK transmitter block diagram. 
Fig. 3

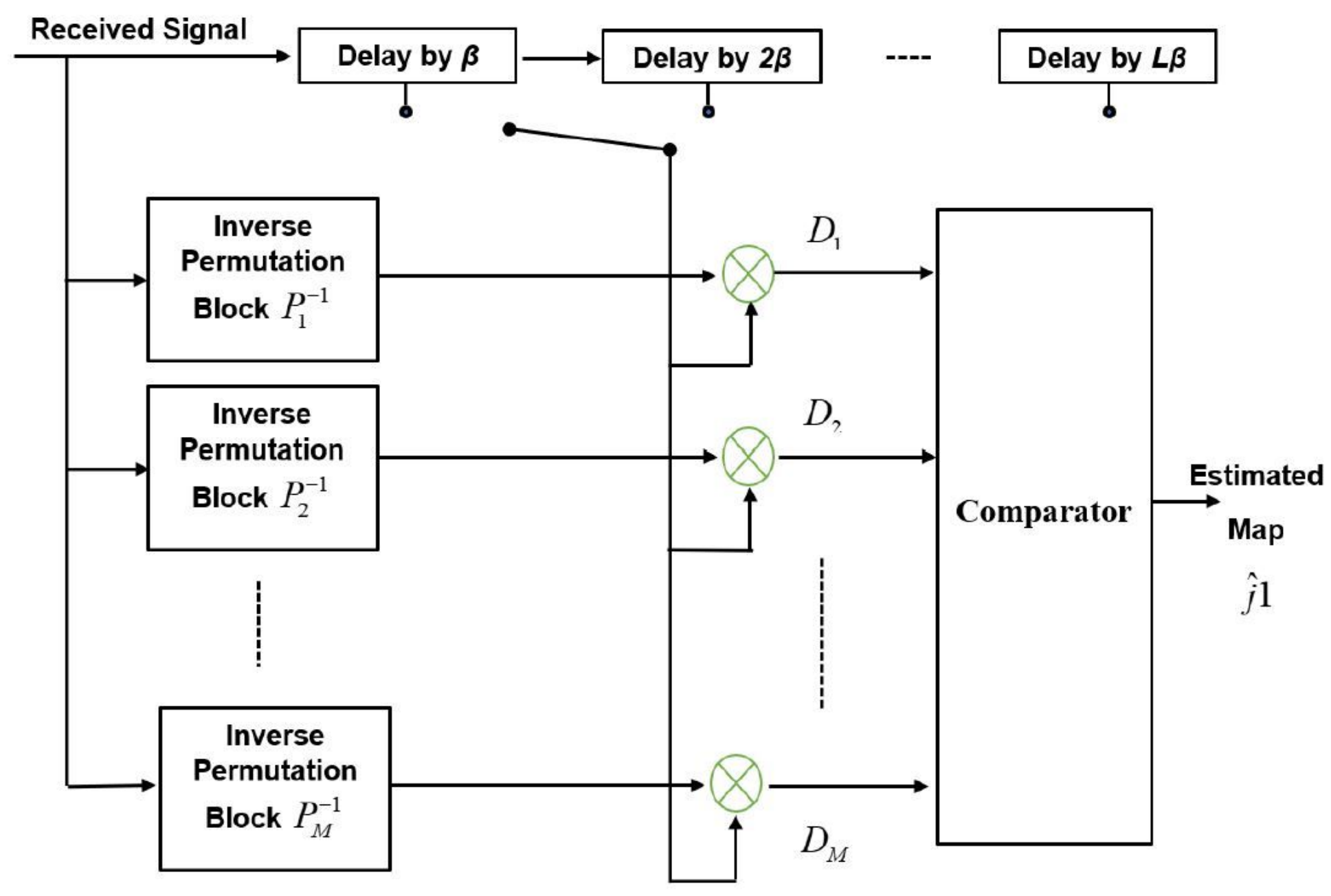

Figure 3

SR-PI-DCSK receiver diagram. 


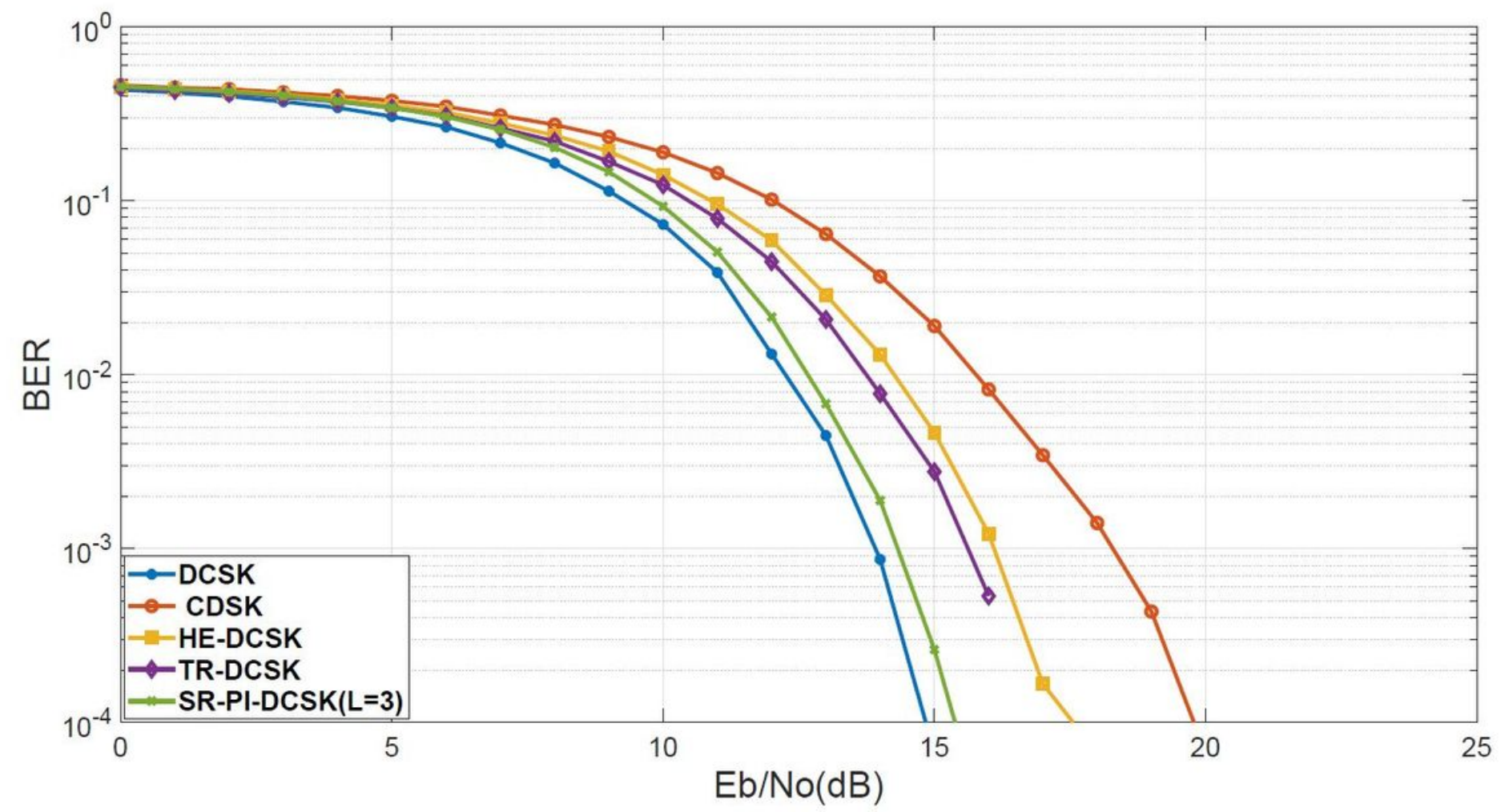

Figure 4

The bit error rate performance of SR-PI-DCSK verses DCSK, CDSK, HE-DCSK, and TR-DCSK for $\beta=100$.

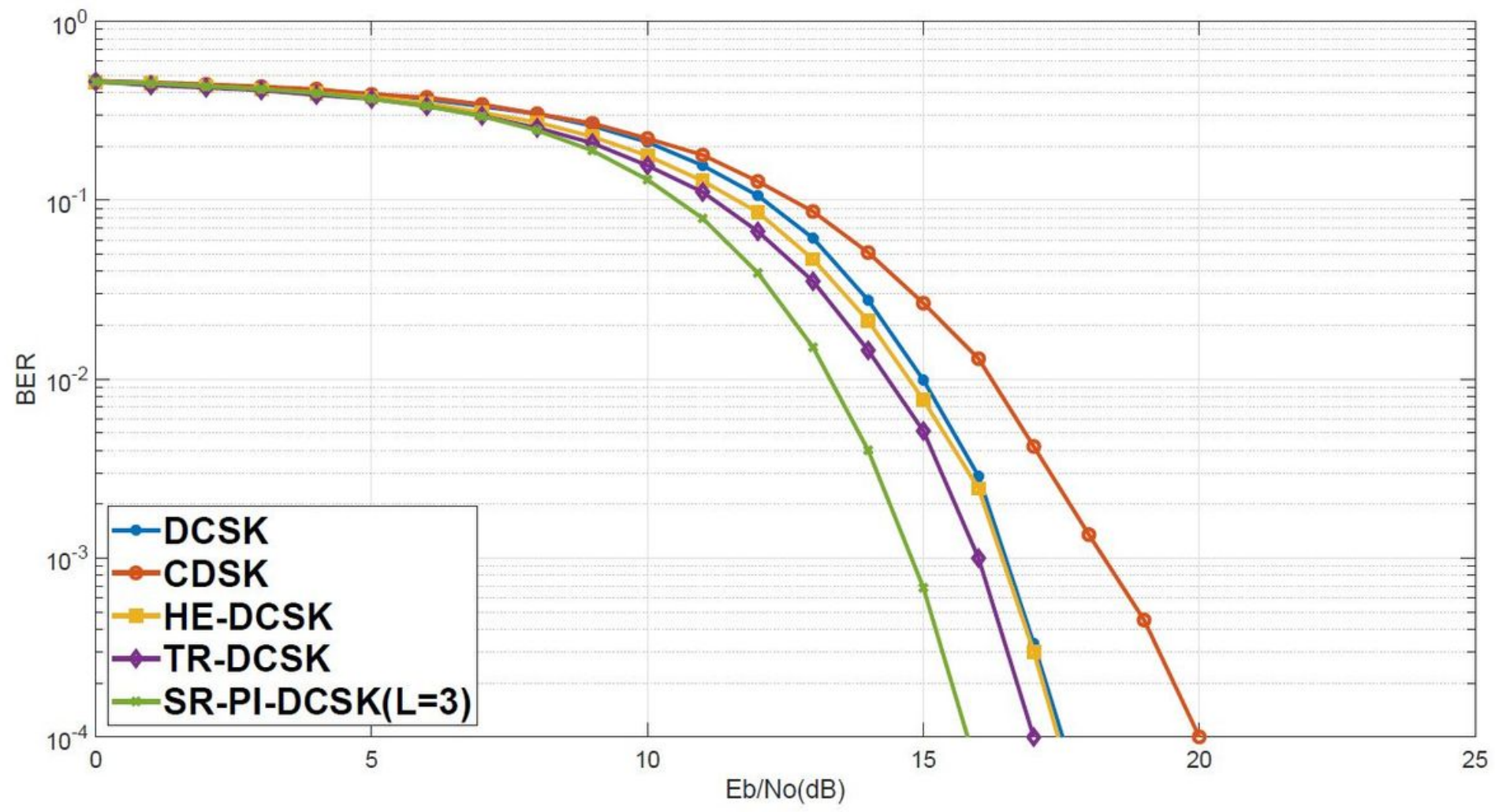

Figure 5 
The bit error rate performance of SR-PI-DCSK verses DCSK, CDSK, HE-DCSK, and TR-DCSK for $\beta=150$.

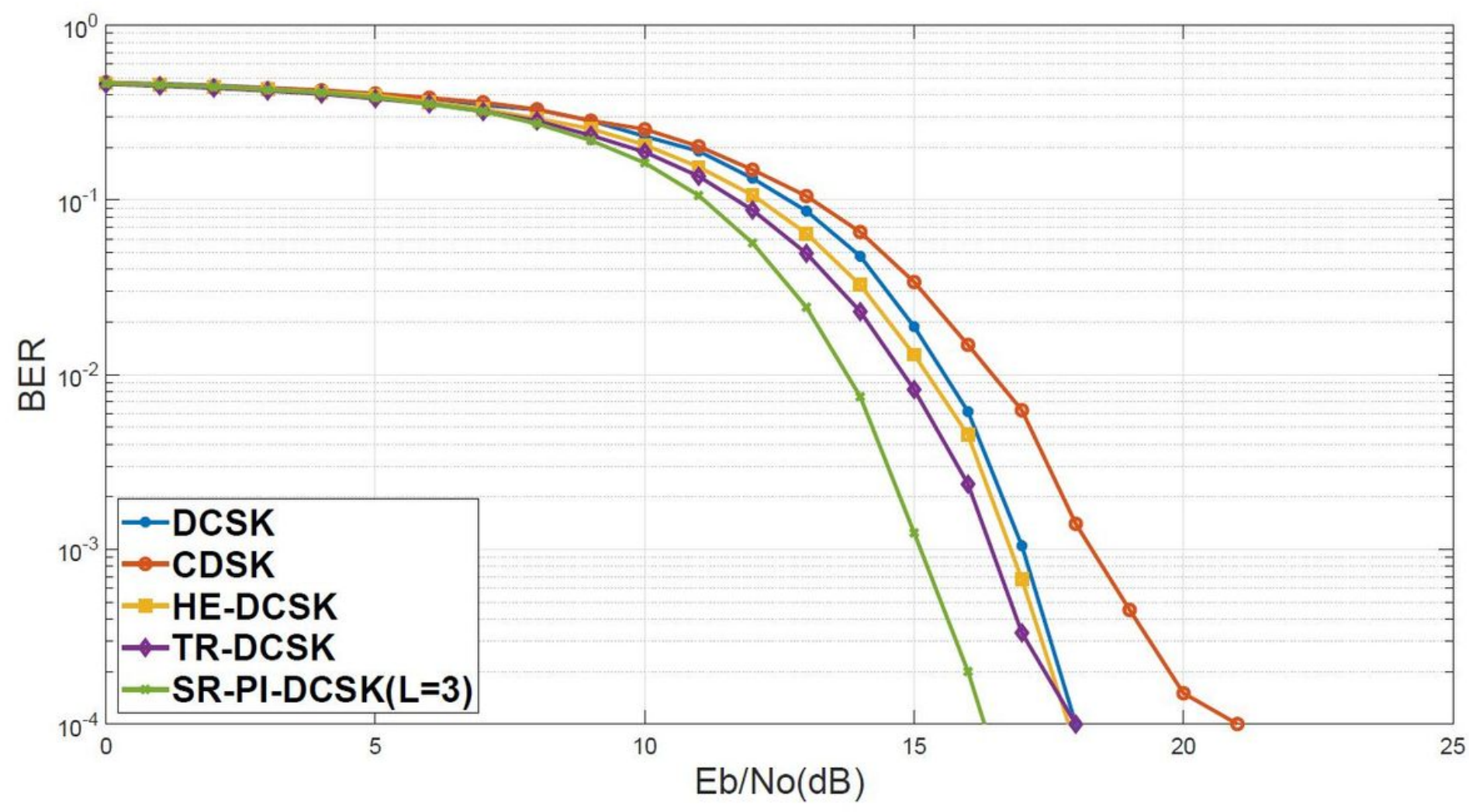

Figure 6

The bit error rate performance of SR-PI-DCSK verses DCSK, CDSK, HE-DCSK, and TR-DCSK for $\beta=200$.

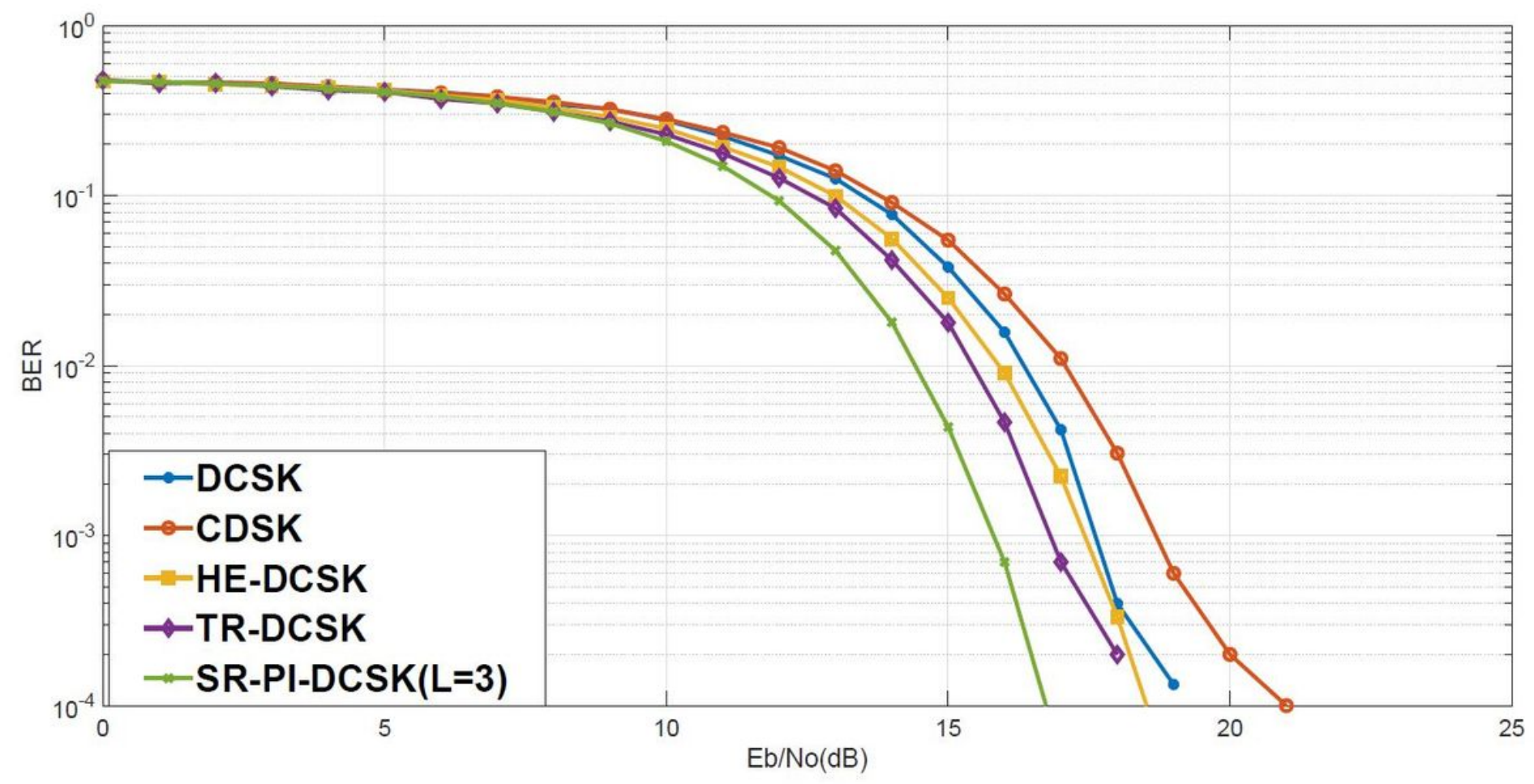

Figure 7 
The bit error rate performance of SR-PI-DCSK verses DCSK, CDSK, HE-DCSK, and TR-DCSK for $\beta=300$.

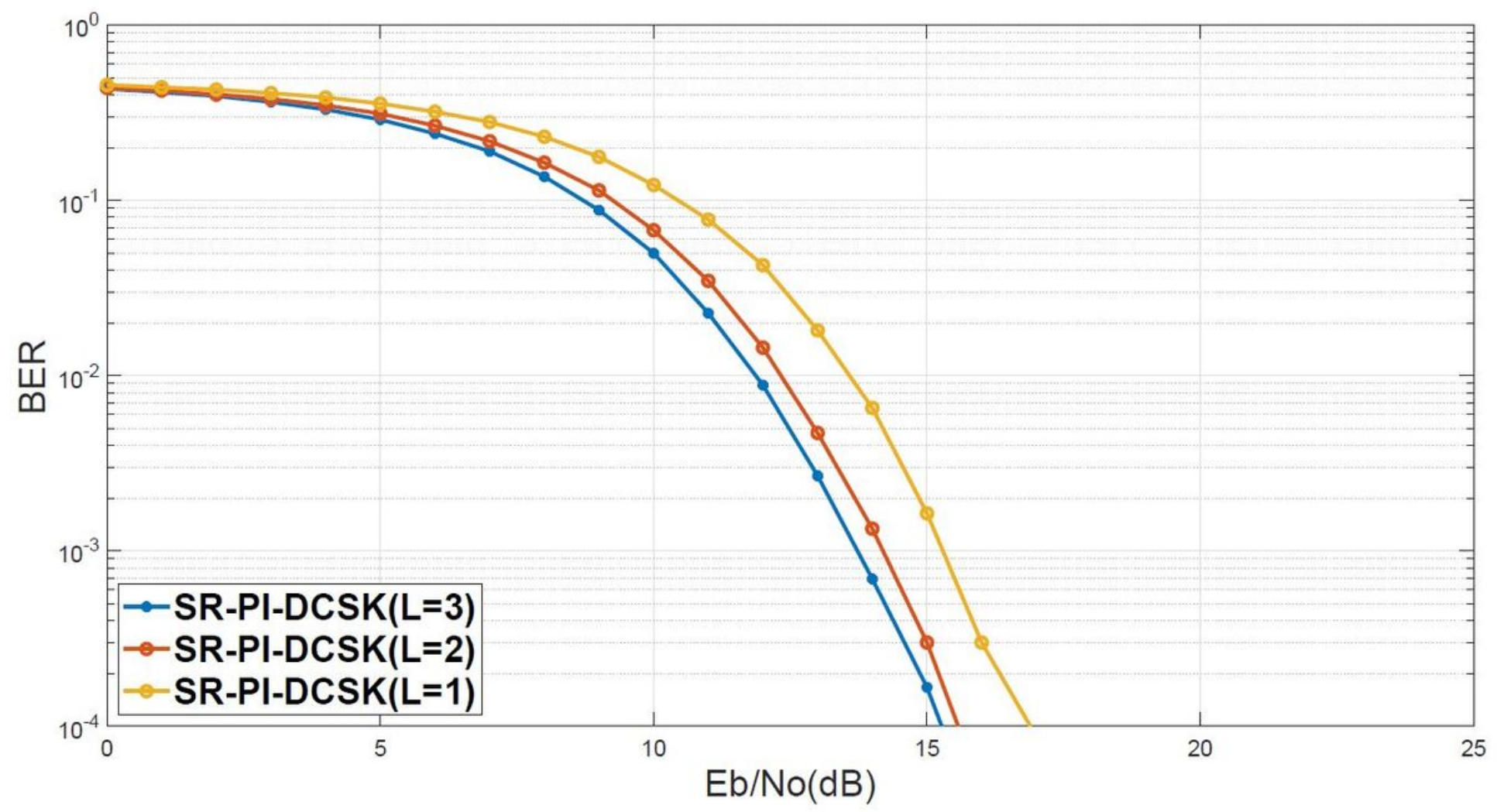

Figure 8

The bit error rate performance at different frame length $(L=1, L=2$ and $L=3)$ for SR-PI-DCSK for $\beta=50$.

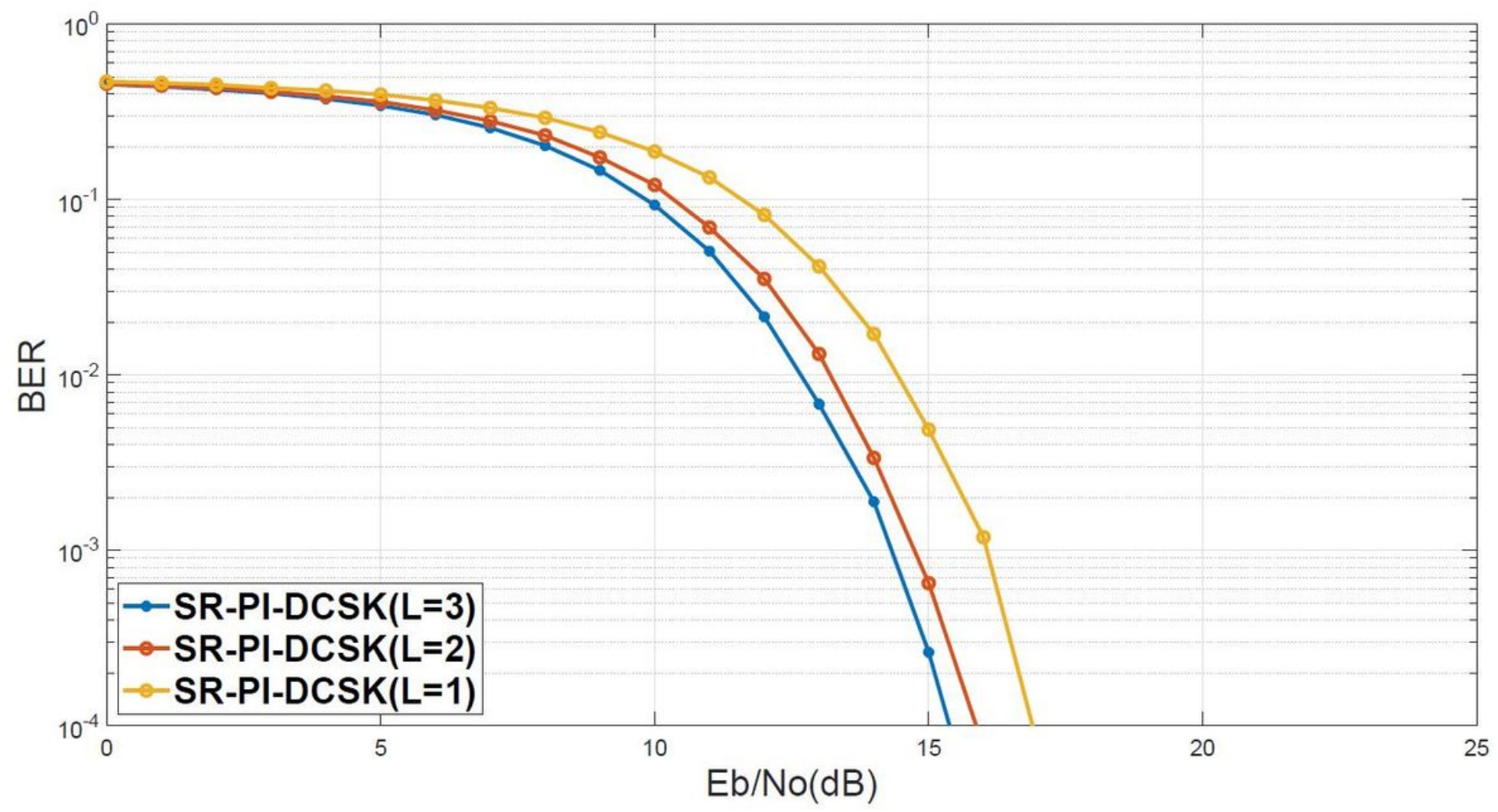




\section{Figure 9}

The bit error rate performance at different frame length $(L=1, L=2$ and $L=3)$ for SR-PI-DCSK for $\beta=$ 100.

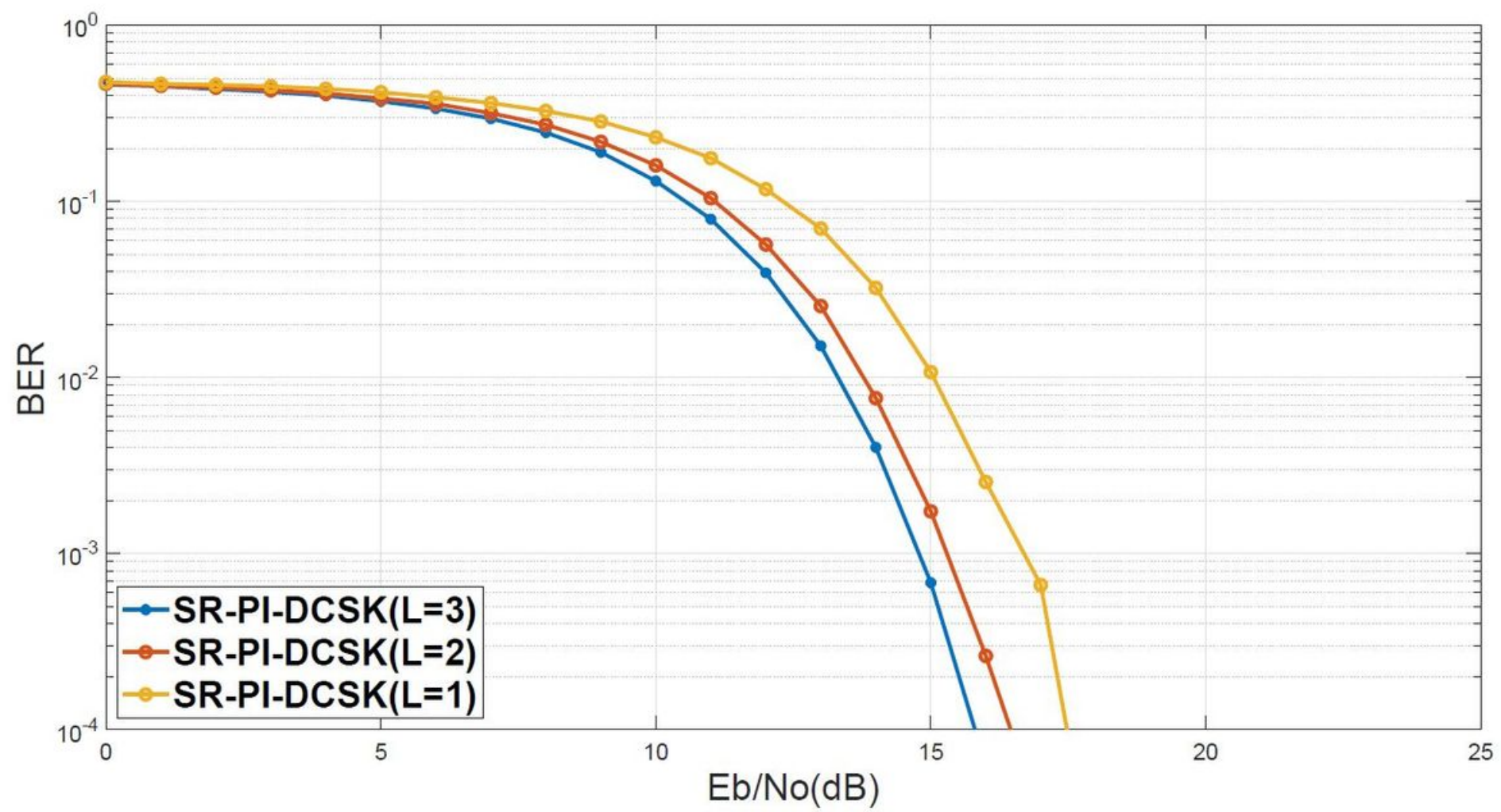

Figure 10

The bit error rate performance at different frame length $(L=1, L=2$ and $L=3)$ for SR-PI-DCSK for $\beta=$ 150. 


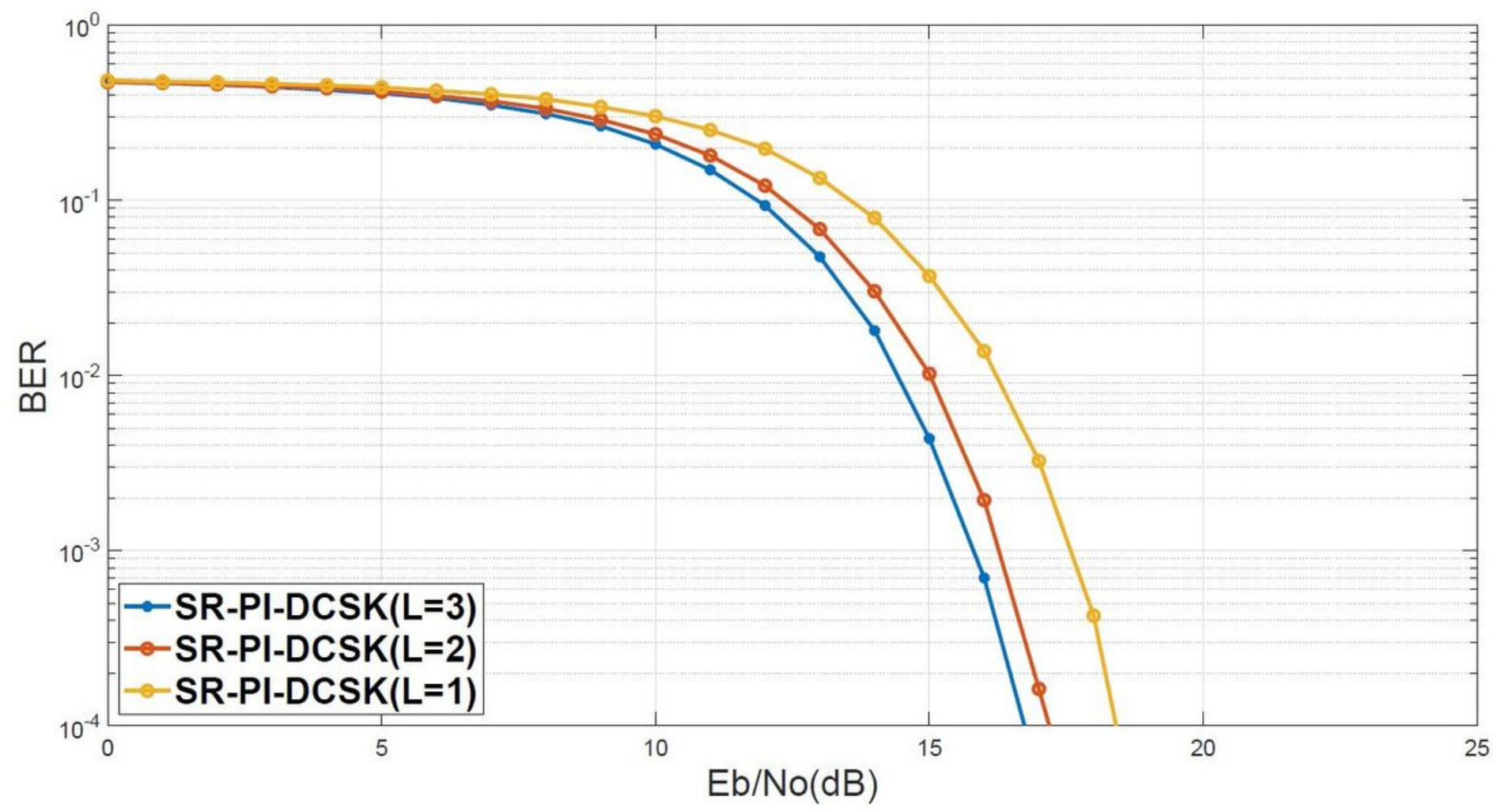

Figure 11

The bit error rate performance at different frame length $(L=1, L=2$ and $L=3)$ for SR-PI-DCSK for $\beta=$ 300 .

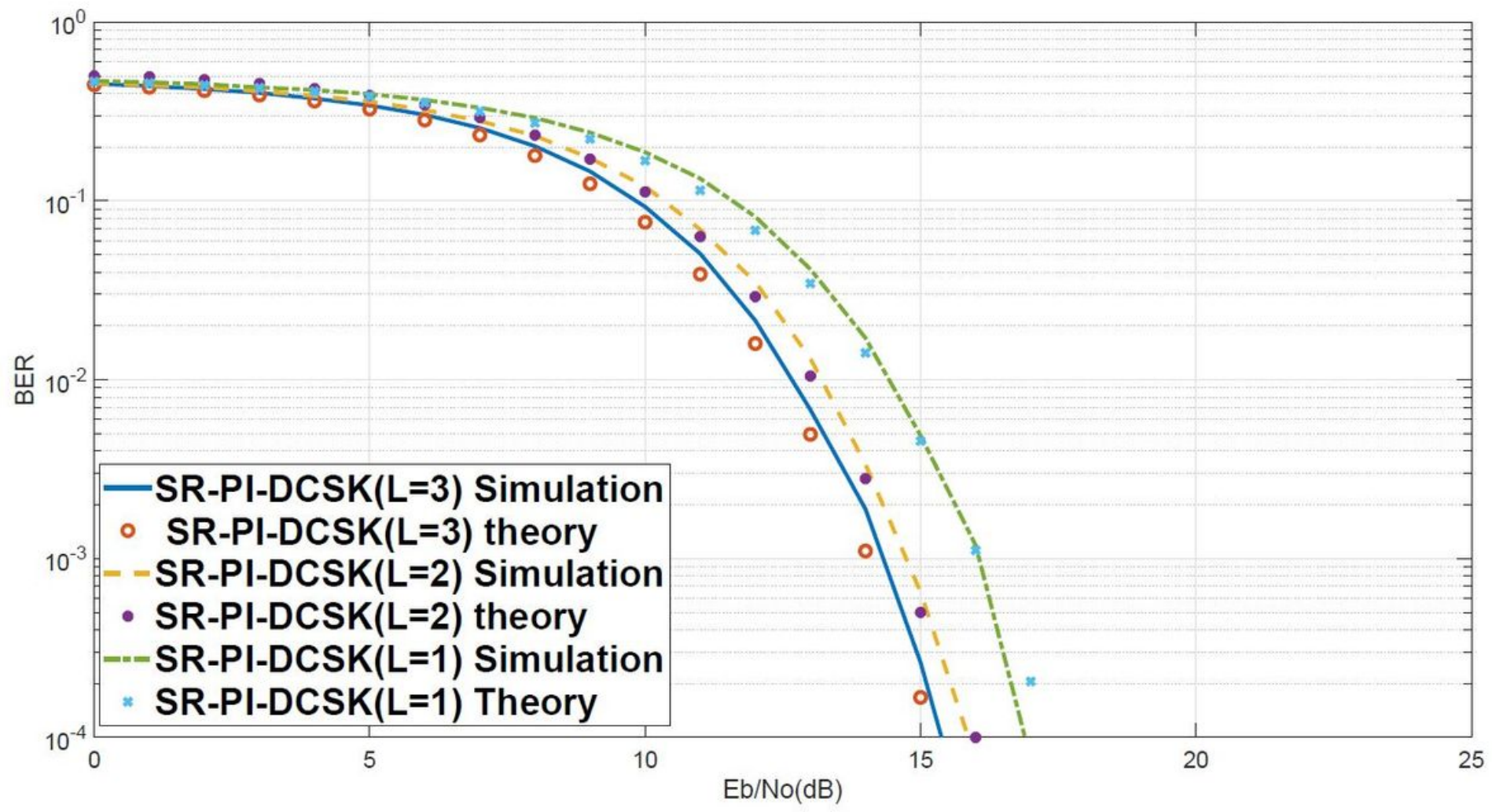

Figure 12 
Theoretical and simulation bit error performance for SR-PI-DCSK for different frame length for $\beta=100$. Simulation results are depicted by lines while theoretical results are depicted by markers.

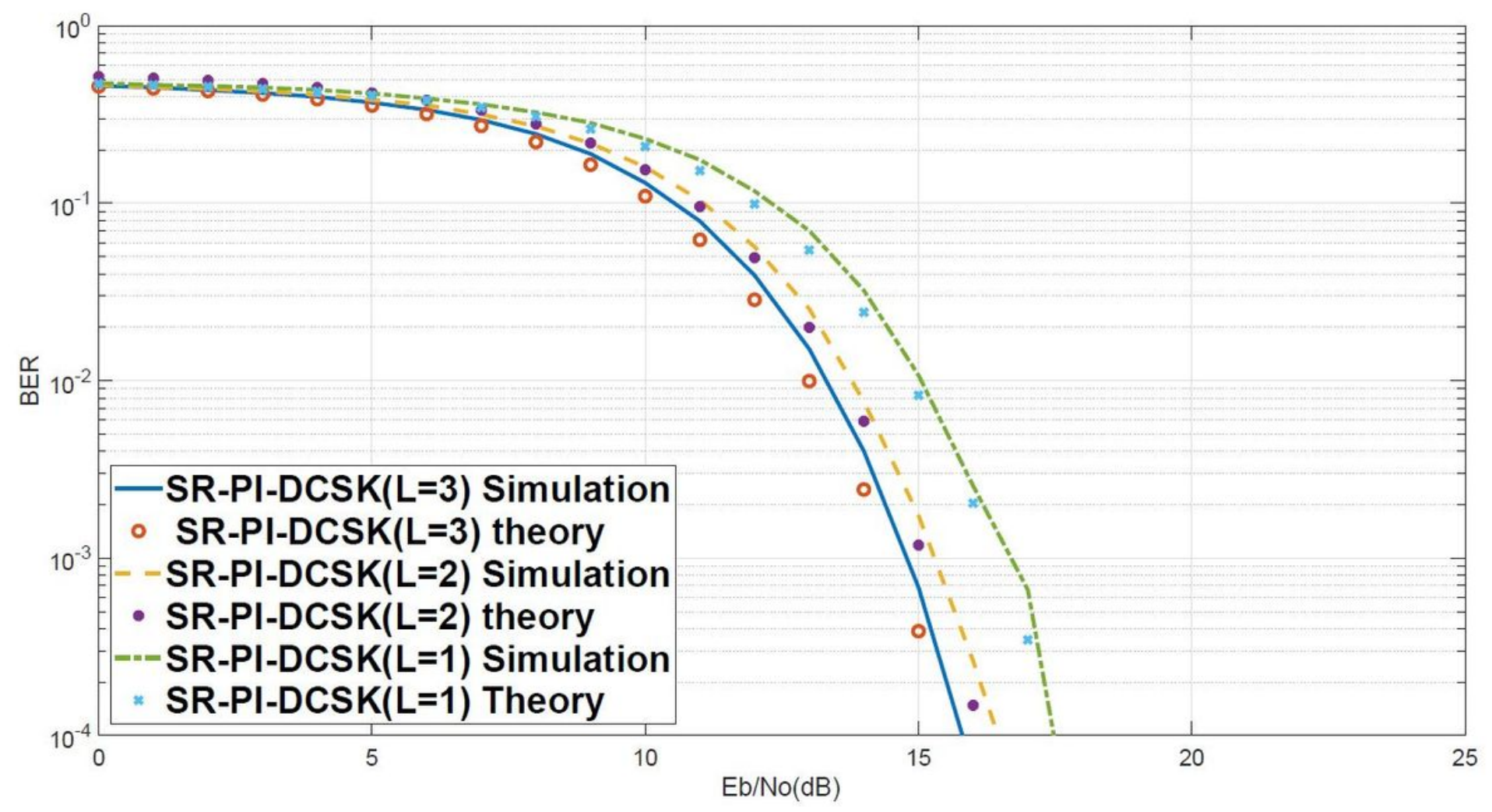

Figure 13

Theoretical and simulation bit error performance for SR-PI-DCSK for different frame length for $\beta=150$. Simulation results are depicted by lines while theoretical results are depicted by markers. 


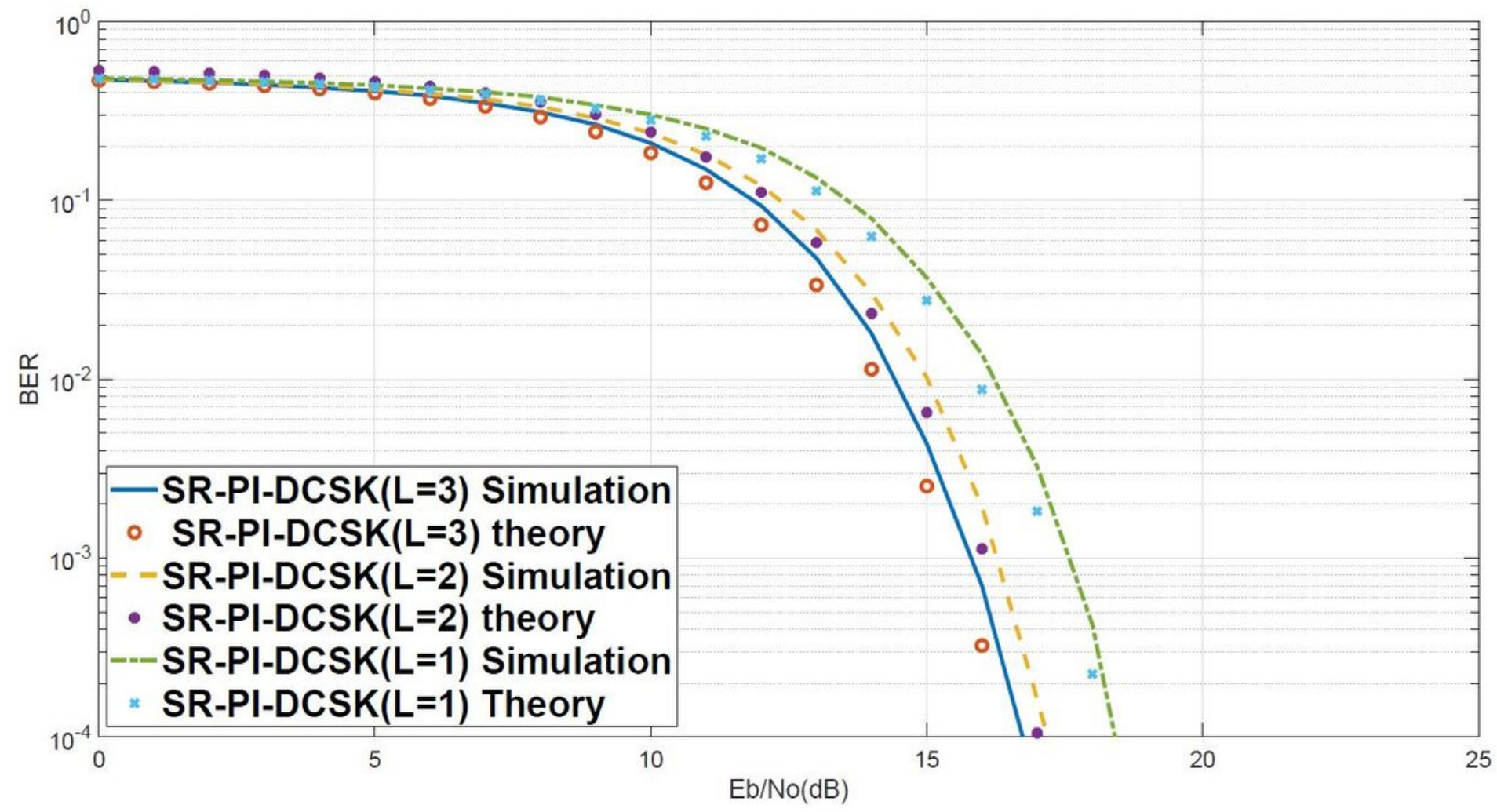

Figure 14

Theoretical and simulation bit error performance for SR-PI-DCSK for different frame length for $\beta=300$. Simulation results are depicted by lines while theoretical results are depicted by markers.

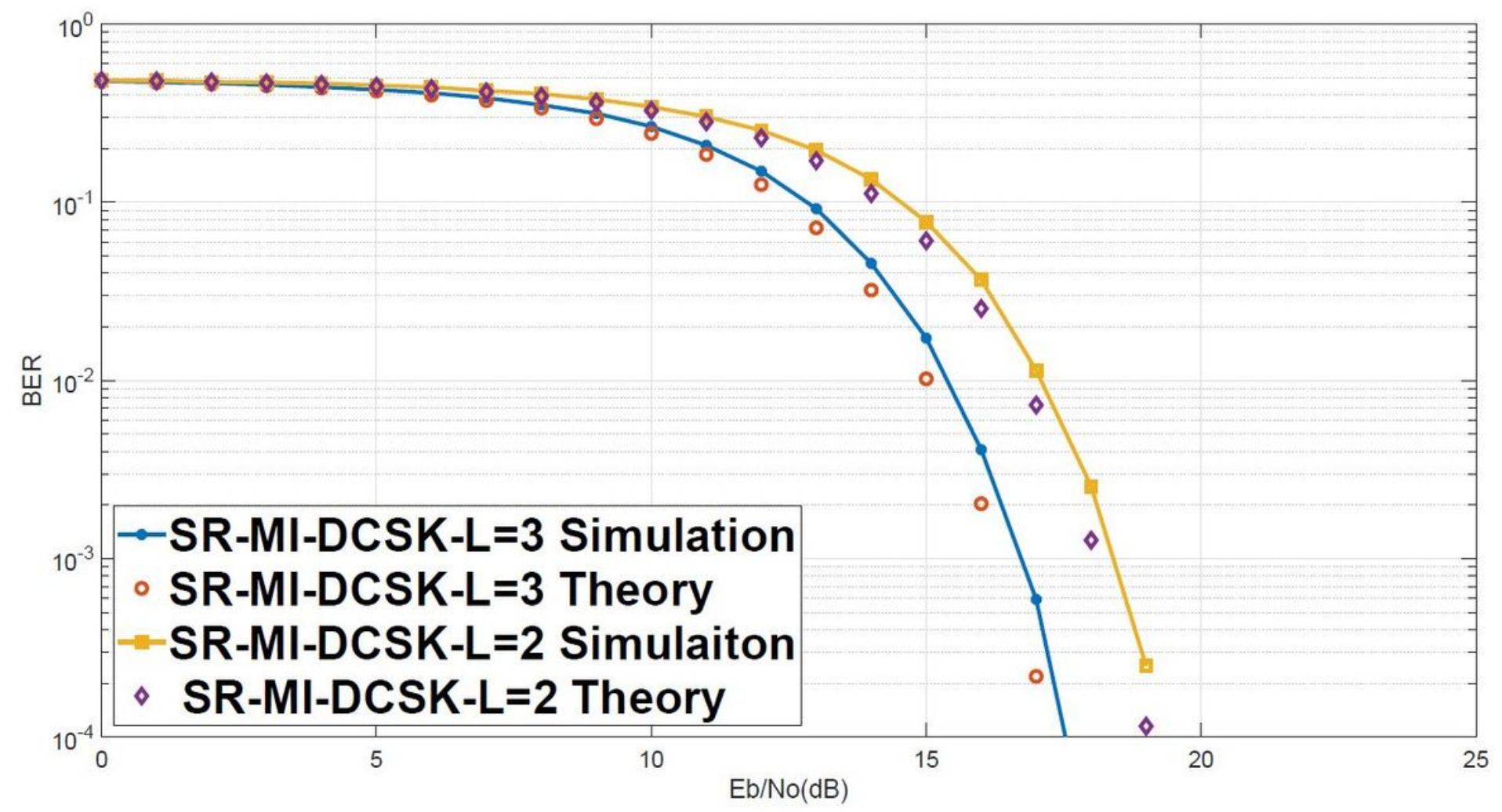

Figure 15 
Theoretical and simulation bit error performance for SR-PI-DCSK for different frame length for $\beta=500$. Simulation results are depicted by lines while theoretical results are depicted by markers.

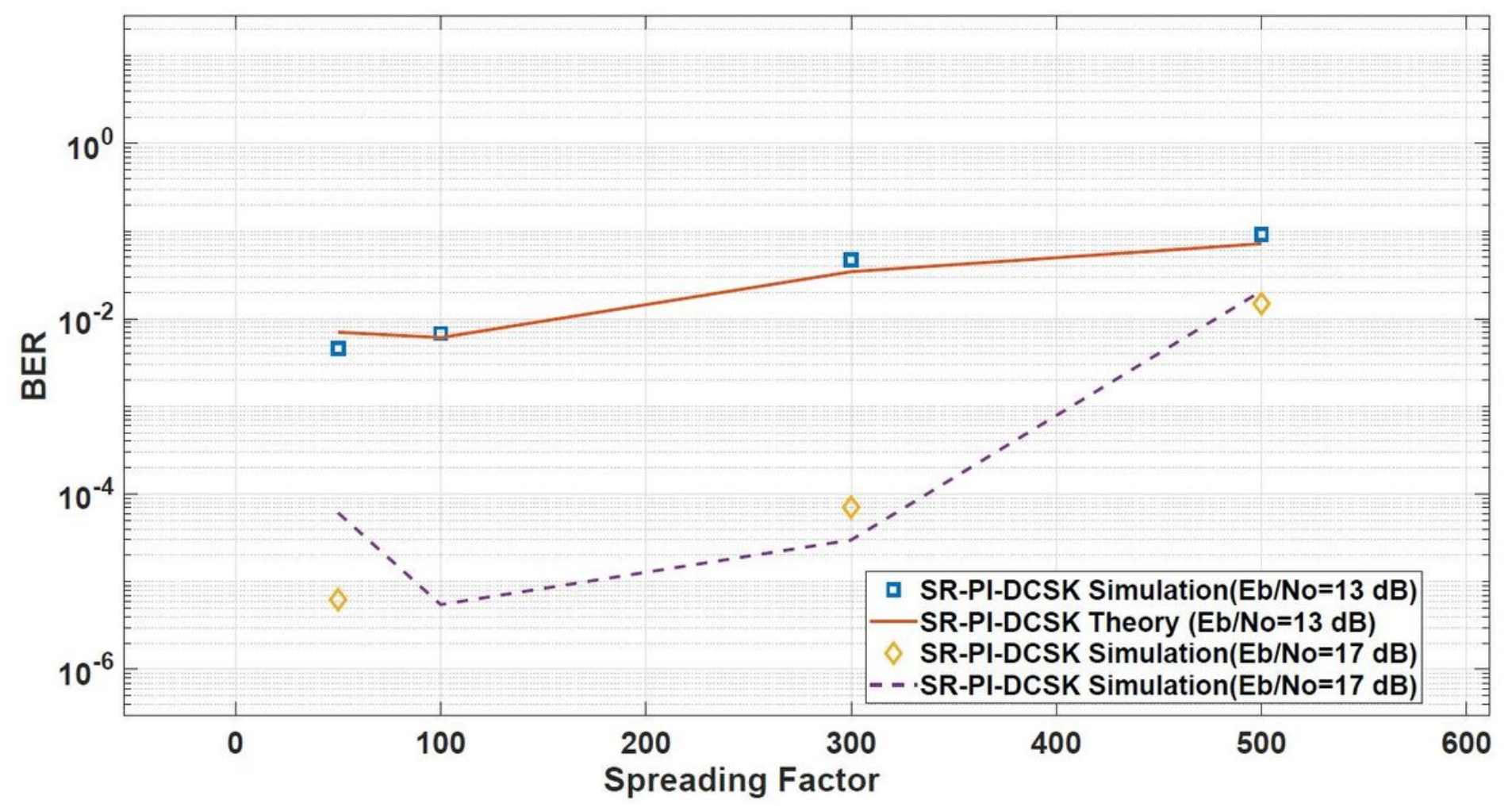

Figure 16

Theoretical and simulation bit error performance for SR-PI-DCSK at different spreading value and L $=3$. 